\title{
The C-terminal CGHC motif of protein disulfide isomerase supports thrombosis
}

\author{
Junsong Zhou, ${ }^{1}$ Yi Wu, ${ }^{1,2}$ Lu Wang, ${ }^{2}$ Lubica Rauova, ${ }^{3}$ Vincent M. Hayes, ${ }^{3}$ Mortimer Poncz, ${ }^{3}$ and David W. Essex ${ }^{2}$ \\ ${ }^{1}$ Cyrus Tang Hematology Center, Jiangsu Institute of Hematology, Collaborative Innovation Center of Hematology, Soochow University, Suzhou, China. ${ }^{2}$ Sol Sherry Thrombosis Research Center, Division of \\ Hematology, Department of Medicine, Temple University School of Medicine, Philadelphia, Pennsylvania, USA. ºivision of Hematology, Children's Hospital of Philadelphia, Philadelphia, Pennsylvania, USA
}

\begin{abstract}
Protein disulfide isomerase (PDI) has two distinct CGHC redox-active sites; however, the contribution of these sites during different physiologic reactions, including thrombosis, is unknown. Here, we evaluated the role of PDI and redox-active sites of PDI in thrombosis by generating mice with blood cells and vessel wall cells lacking PDI ( $M \times 1-C r e ~ P d i f / f l$ mice) and transgenic mice harboring PDI that lacks a functional C-terminal CCHC motif [PDI(ss-0o) mice]. Both mouse models showed decreased fibrin deposition and platelet accumulation in laser-induced cremaster arteriole injury, and PDI(ss-0o) mice had attenuated platelet accumulation in $\mathrm{FeCl}_{3}$-induced mesenteric arterial injury. These defects were rescued by infusion of recombinant PDI containing only a functional C-terminal CCHC motif [PDI(00-ss)]. PDI infusion restored fibrin formation, but not platelet accumulation, in eptifibatide-treated wild-type mice, suggesting a direct role of PDI in coagulation. In vitro aggregation of platelets from PDI(ss-00) mice and PDI-null platelets was reduced; however, this defect was rescued by recombinant PDI(0o-ss). In human platelets, recombinant PDI(ss-0o) inhibited aggregation, while recombinant PDI(oo-ss) potentiated aggregation. Platelet secretion assays demonstrated that the C-terminal CCHC motif of PDI is important for P-selectin expression and ATP secretion through a non- $\alpha$ llb $\beta 3$ substrate. In summary, our results indicate that the C-terminal CCHC motif of PDI is important for platelet function and coagulation.
\end{abstract}

\section{Introduction}

Protein disulfide isomerase (PDI) is the prototypic member of the PDI family of enzymes that catalyze the reversible formation and isomerization of disulfide bonds (1). Platelet PDI mediates platelet aggregation, secretion $(2,3)$, and adhesion of human platelets (4, 5). Mouse platelets lacking PDI have decreased aggregation, and mice with PDI-deficient platelets have decreased platelet accumulation at a site of vascular injury; however, fibrin deposition was normal (6). The role of PDI in coagulation and particularly in the regulation of tissue factor activity has been unclear $(7,8)$. In vitro studies originally found PDI to be a negative regulator of tissue factor activation in cells (9); however, a subsequent report failed to confirm this and instead reported that phosphatidylserine exposure on cells was the principal factor regulating tissue factor activation (10). Depending on the specific conditions and redox environment, PDI may potentiate or inhibit coagulation $(11,12)$ or tissue factor activation $(8,9,12-14)$. PDI has been shown to have a role in vivo in fibrin generation $(13,15-17)$, although some of these studies used PDI inhibitors of uncertain specificity $(6,18)$.

It has been known for several decades that PDI has two redox -active CGHC active site found in the catalytic a and a' domains near the $\mathrm{N}$ - and C-terminus, respectively. Previous in vitro studies demonstrated that both CGHC sequences of PDI had approximately equal catalytic activity against the substrates scrambled RNase or insulin (19). On the other hand, functional nonequiva-

Authorship note: Junsong Zhou and Yi Wu contributed equally to this work. Conflict of interest: The authors have declared that no conflict of interest exists. Submitted: January 6, 2015; Accepted: September 28, 2015.

Reference information: J Clin Invest. 2015;125(12):4391-4406. doi:10.1172/JCI80319. lence of each CGHC active site of PDI was demonstrated against reduced denatured RNase (20). Specifically, inactivation of the $\mathrm{N}$-terminal active site decreased the Kcat of PDI, while inactivation of the $\mathrm{C}$-terminal active site increased the $\mathrm{Km}$. Using a 28-residue synthetic peptide based on bovine pancreatic trypsin inhibitor (BPTI) as a substrate, the C-terminal active site was somewhat better at rearrangement of the disulfide bond $30-51$, while the $\mathrm{N}$-terminal active site was better at rearrangement of the disulfide bond 5-55 (21). Using an in vitro assay that reconstituted the human Ero1-L $\alpha /$ PDI oxidative folding pathway, the C-terminal active site of PDI was found to be much more active than the $\mathrm{N}$-terminal active site (22). The N-terminal active site of yeast PDI (29\% identity to human PDI) was more important than the C-terminal active site against the yeast PDI substrate procarboxypeptidase (23). These studies indicate distinct functions for the active sites of PDI. However, little or nothing is known about the relative importance of the active sites of PDI in mammalian cells or in proteins proven to be physiologic substrates $(24,25)$.

The PDI-catalyzed reactions in the above studies involve isomerase activity (scrambled RNase, ref. 19; and BPTI assays, ref. 21) and oxidase activity $(20,22)$. PDI on the platelet surface catalyzes reduction of disulfide bonds (26-28) and may also catalyze isomerization $(29,30)$ and oxidation (31) reactions. PDI on the platelet surface also catalyzes release of nitric oxide from S-nitrosoglutathione $(32,33)$.

PDI facilitates activation of the $\alpha \operatorname{IIb} \beta 3$ fibrinogen receptor on the platelet surface $(3,6,34)$. However, the roles of each CGHC active site in this process are unknown. Inhibition of PDI is reported to attenuate platelet aggregation $(3,34)$, and inhibitors of PDI have been proposed as antithrombotic agents (35). 
Knowledge of the role of PDI in thrombosis and the mechanism by which it acts would facilitate the design of therapeutic inhibitors.

Another member of the PDI family of enzymes, ERp57, with high homology to PDI, is also needed for platelet accumulation and fibrin generation $(36,37)$. Although little is known about the interactions between PDI and ERp57 in thrombosis, they play distinct roles in polyomavirus infection (38). Specifically, PDI reduces disulfide bonds in the polyomavirus protein, while ERp57 isomerizes disulfide bonds. While ERp57 and PDI appear to have distinct roles in thrombosis $(36,37)$, how they cooperate in thrombosis is not known.

The aim of this study was to determine the relative importance of each active site of PDI in platelet function and coagulation and to characterize the role of PDI in fibrin generation in vivo without the use of inhibitors. To do this, we generated Pf4-Cre and Mx1-Cre Pdi knockout mice and knockout transgenic mice with the C-terminal CGHC motif of PDI rendered inactive [transgenic PDI(ss-oo) mice]. We found that the C-terminal CGHC motif of intravascular PDI was required for both platelet accumulation and fibrin generation.

\section{Results}

Targeted knockout of Pdi in mouse platelets. Since global deletion of PDI is embryonically lethal in mice, to determine whether platelet-derived PDI had a role in thrombus formation, we generated a platelet-specific knockout. Conditional Pdi knockout mice were generated by introducing loxP sites flanking the ATG start codon and coding exons 1 and 2 of the Pdi gene by gene targeting (Figure 1A). Using a 5 ' cDNA probe, wild-typegenomic DNA yielded a 5.1-kb fragment, while a Flp-mediated excised allele yielded an $8.1-\mathrm{kb}$ hybridization signal. Targeting of the PDI locus was confirmed by Southern blot of PciI-digested genomic DNA hybridized with this probe (Figure 1B). To generate Pf4-Cre Pdit/fl mice, homozygous $P d i^{f l f l}$ mice on a mixed C57BL/6 $\times 129 \mathrm{~Sv}$ genetic background were crossed with Pf4-Cre mice (39) on a C57BL/6 background. To generate platelet-specific Pdi knockout mice and experimental control mice, $P f 4$-Cre Pdillfl mice were mated with $P d i^{f l / f l}$ mice, which produced $P f 4$-Cre $P d i^{f / f l}$ mice and $P d i^{l / f l}$ littermate controls.

As detected by RT-PCR, the mRNA for Pdi was absent from platelets of Pf4-Cre Pdillfl mice (Figure 1C). PDI was absent in Western blots of platelet lysates prepared from $\mathrm{Pf} 4$-Cre Pdil/fl mice, confirming successful targeting of the enzyme (Figure 1D). No changes in ERp57, ERp5, or ERp72 levels were detected by Western blotting of PDI-deficient platelets (Figure 1D). Complete blood counts in Pf4-Cre Pdil/fl mice revealed no abnormalities. Platelet counts (Figure $1 \mathrm{E}$ ) and size (mean platelet volume $[\mathrm{MPV}]=6.59 \pm 0.02 \mathrm{fl} \mathrm{SEM}$ ) were comparable to those of $\mathrm{Cre}^{-}$littermates $(\mathrm{MPV}=6.94 \pm 0.14 \mathrm{fl}$, $P=\mathrm{NS}, n=10$, per arm). There were no differences in expression of the major platelet surface glycoproteins, $\alpha \mathrm{IIb} \beta 3$, GpIb $\alpha$, and GpVI (Figure 1F). Since platelet counts, expression of major platelet surface glycoproteins, and MPV were normal in Pf4-Cre Pdifl/fl mice, either PDI does not have a significant role in platelet production or biogenesis of the major glycoproteins or other members of the PDI family compensate for loss of PDI $(25,40)$.

Having generated mice with PDI-deficient platelets, we were able to further test PDI inhibitors for their specificity. A commonly used inhibitory antibody to PDI, RL90, inhibited aggregation of
PDI-deficient platelets in a dose-dependent manner (Supplemental Figure 1, A and B; supplemental material available online with this article; doi:10.1172/JCI80319DS1). Another inhibitor of PDI, quercetin-3-rutinoside, which also inhibits aggregation of PDIdeficient platelets (6), inhibited ERp57 in the Di-E-GSSG assay as effectively as it inhibited PDI (Supplemental Figure 1, C and D). Since both RL90 and quercetin-3-rutinoside inhibit aggregation of PDI-null platelets (6) and ERp57 (41), studies using targeted Pdi knockout mice and transgenic mice are important in characterizing the role of PDI in vivo.

Mice with platelets lacking PDI have long tail bleeding times and times to occlusion and impaired platelet function. Tail bleeding times were approximately doubled in mice with PDI-deficient platelets relative to those in $\mathrm{Cre}^{-}$littermate controls (Figure 2A). The time to complete occlusion after the $\mathrm{FeCl}_{3}$-induced injury of the carotid artery was also doubled in the mice with PDI-deficient platelets (Figure 2B). These findings indicate that platelet-derived PDI is required for both hemostasis (tail bleeding times) and thrombosis (carotid artery occlusion). Thrombin-induced activation of $\alpha \operatorname{IIb} \beta 3$ (Figure 2C), P selectin expression (Figure 2D), and thrombin- and convulxin-induced aggregation (Figure 2, E and F) were decreased in platelets lacking PDI. Higher doses of thrombin or convulxin overcame the aggregation defect (Supplemental Figure 2).

The catalytic activity of the C-terminal CGHC motif of PDI is required for platelet aggregation. Recombinant wild-type PDI [rPDI(ss-ss)] potentiated aggregation of human platelets, while completely inactivated recombinant PDI [rPDI(oo-oo)] inhibited aggregation (Figure 3A). This indicates that the catalytic activity of the active sites of PDI is required for PDI function. To determine the relative importance of the two active sites of PDI in platelet function, we tested the effect of two mutants of the enzyme on aggregation. One mutant had the $\mathrm{N}$-terminal CGHC motif inactivated, rPDI(oo-ss), and the other mutant had the C-terminal CGHC motif inactivated, rPDI(ss-oo). The mutant that retained the C-terminal CGHC motif potentiated thrombin-induced aggregation to a similar degree as wild-type rPDI(ss-ss) (Figure 3A). In contrast, the mutant with the inactive C-terminal CGHC motif inhibited aggregation. These results imply that the C-terminal active site of PDI is important for platelet aggregation. While rPDI(ss-oo) inhibited platelet aggregation, the extent of the inhibition was not as great as that seen with rPDI(oo-oo) (Figure 3A), suggesting that the $\mathrm{N}$-terminal active site may have some contribution to platelet aggregation. Preincubating PDI-null platelets with rPDI(oo-ss) led to a dose-dependent recovery of platelet aggregation (Figure 3B), consistent with the importance of the catalytic activity of the C-terminal CGHC motif of PDI to human platelet biology.

Characterization of knockout transgenic PDI(ss-oo) mice. We then went on to determine the physiological significance of the two CGHC active sites individually in vivo; however, while we could establish transgenic PDI(ss-oo) mice and rescue the lethal phenotype of $\mathrm{Pdi}^{-/-}$mice, a parallel transgenic mouse PDI(oo-ss) with an inactive N-terminal CGHC motif and an active C-terminal CGHC motif could not rescue the lethal $\mathrm{Pdi}^{-/-}$phenotype. This suggests that the $\mathrm{N}$-terminal but not $\mathrm{C}$-terminal CGHC site is required for animal viability (which is opposite to the findings of the hemostasis studies shown later). 
A

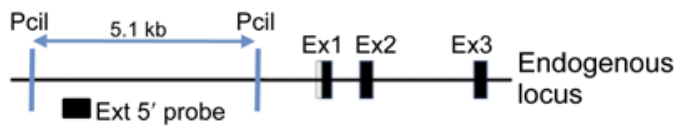

Pcil
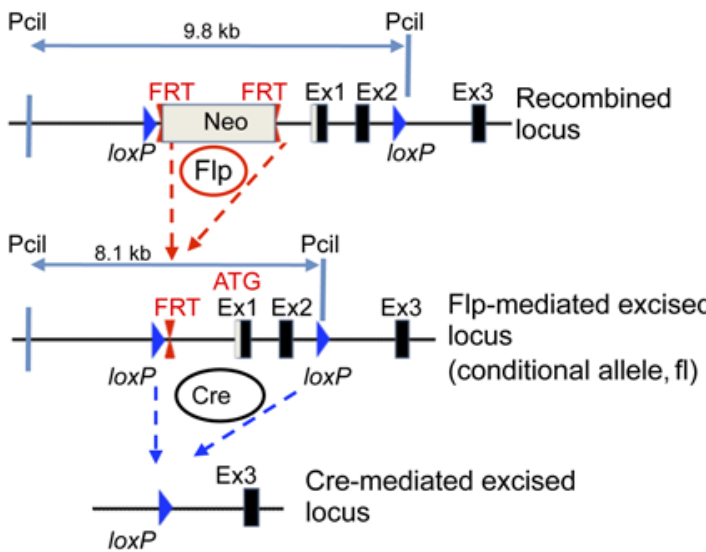

B

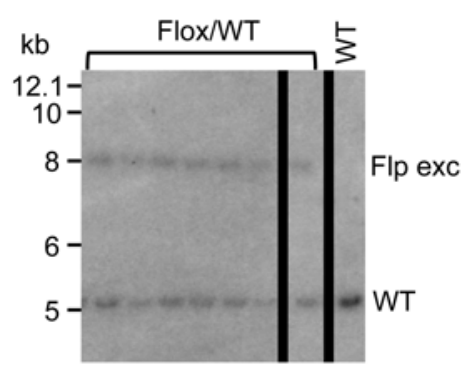

C

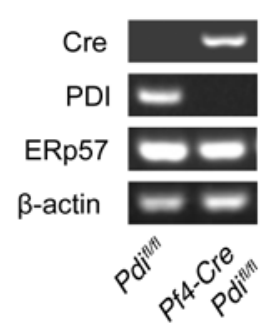

D

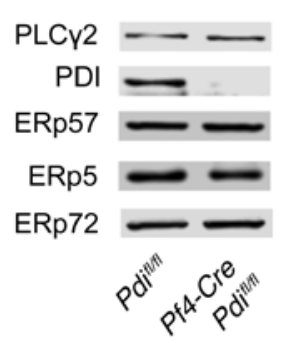

E

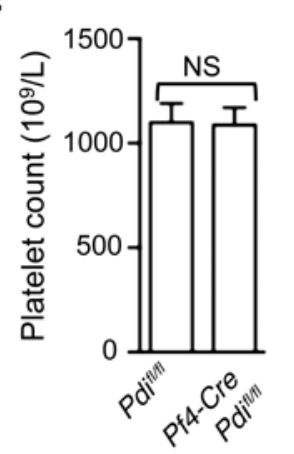

$\mathbf{F}$

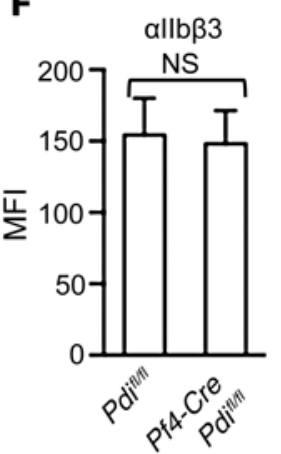

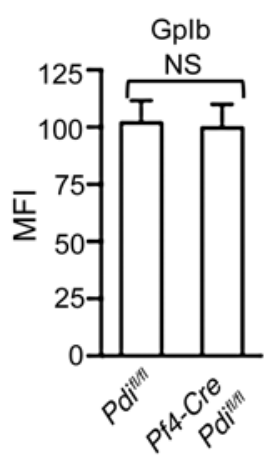

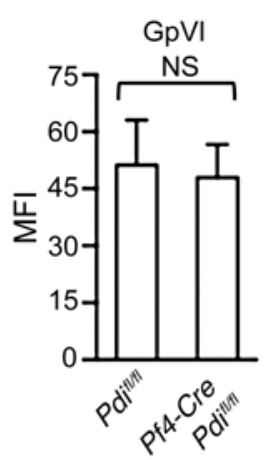

Figure 1. Deletion of $P d i$ in platelets. (A) Scheme of the targeting strategy. Homologous recombination of the Pdi conditional targeting vector into the Pdi gene in embryonic stem cells introduced 2 loxP sites (triangles) upstream of exon 1 and the ATC start codon and downstream of exon 2 and 2 FRT sites (surrounding the Neo cassette that was used for selection). The targeted neo cassette allele (recombined locus) was removed by Flp-mediated recombination of FRT sites (to form the conditional allele, $\mathrm{fl}$ ). Pf4-Cre recombinase (Cre) expressed with the $P$ di conditional allele deletes exons $1-2$, generating the $P d i$-deleted allele. (B) Southern blot analysis of heterozygous Flp-excised Pdi-floxed mice. The Pcildigested mouse genomic DNAs (digestion sites indicated in $\mathbf{A}$ ) were analyzed by hybridization with the external $5^{\prime}$ probe (ext 5' probe, A). The wild-type allele with the Pdi endogenous locus (WT) and the Flp-mediated excised allele (Flp exc) gave rise to $5.1-\mathrm{kb}$ and 8.1-kb hybridization bands, respectively. (C-F) Characterization of platelets of Pf4-Cre Pdifl/fl mice. (C) Platelet mRNA levels were evaluated by RT-PCR to demonstrate the absence of Pdi mRNA. (D) Western blots of lysates using a polyclonal rabbit anti-PDI antibody and antibodies against ERp57, ERp5, and ERp72. The PLC $\gamma 2$ loading controls for PDI are shown. Separate loading controls were run for ERp57, ERp5, and ERp72 with similar amounts of protein found in each sample (data not shown). Blots in $\mathbf{C}$ and $\mathbf{D}$ are representative of 3 separate experiments. (E) Comparison of platelet counts between $P d i^{f / f f l}$ and Pf4-Cre Pdiflffl mice; mean \pm SEM, $n=10, t$ test. (F) Glycoprotein ( $\alpha$ llb $\beta 3$, GPIb, or GPVI) expression on platelets from Pf4-Cre Pdifl/fl and Pdiflffl littermate controls was analyzed by flow cytometry; mean \pm $\mathrm{SEM}, n=10, t$ test. MFI, mean FI.
PCR of tail DNA indicated that the transgenic mice expressed the transgene (Figure 4, A and B). The mRNA levels of the transgene were comparable to those of endogenous Pdi in wild-type control mice on the same mixed C57BL/6 $\times 129$ Sv genetic background (Figure $4 \mathrm{C}$ ). Sequencing of the PCR product of the cDNA transgene demonstrated the 2 mutations of nucleotide $\mathrm{G}$-to- $\mathrm{C}$ in 2 places, yielding 2 Cys-to-Ser mutations in the PDI(ss-oo) transgene (Figure 4D). The platelets of transgenic and wild-type mice expressed similar PDI protein levels (Figure 4E) and similar ERp57 and ERp72 levels. Levels of PDI, ERp57, and ERp72 in endothelial cells, monocytes, and neutrophils were comparable (Supplemental Figure 3A). Complete blood counts in transgenic PDI(ss-oo) mice revealed no abnormalities. Platelet counts (Figure $4 \mathrm{~F}$ ) and size $(\mathrm{MPV}=6.33 \pm 0.23 \mathrm{fl}$ ) were comparable to those of wild-type controls $(\mathrm{MPV}=6.55 \pm 0.64 \mathrm{fl}, P=\mathrm{NS}, n=12$ mice per arm). There was no difference in expression of $\alpha \mathrm{IIb} \beta 3$, GPIb $\alpha$, or GpVI (Figure $4 G)$. The level of PDI on transgenic platelets was evaluated by flow cytometry using a rabbit anti-PDI antibody. This antibody was specific for PDI on a Western blot of platelet lysates (Supplemental Figure $3 \mathrm{~B}$ ) and did not cross react with other PDIs (Supplemental Figure 3C). Using this antibody, similar levels of PDI were found on transgenic and wild-type platelets in both resting and activated conditions (Supplemental Figure 3, D and E). The levels of plasma PDI in transgenic PDI(ss-oo) mice and wild-type mice were also similar (Supplemental Figure 3F). Since the PDI(ss-oo) transgene is expressed in all mouse tissues, the mutant PDI will be expressed in hepatocytes as well as vascular wall cells. The prothrombin time (PT) and the activated partial thromboplastin time (APTT) of these mice were normal (Supplemental Figure 3G), consistent with these mice not having developed a coagulopathy.

Transgenic PDI(ss-oo) mice have prolonged tail bleeding times and times to occlusion and impaired platelet function. Similar to that in the mice with PDI-deficient platelets, the tail bleeding times were approximately double in transgenic PDI(ss-oo) mice compared with those in wild-type mice (Figure $5 \mathrm{~A}$ ). The times to thrombosis after $\mathrm{FeCl}_{3}$-induced injury of the carotid artery were also prolonged 

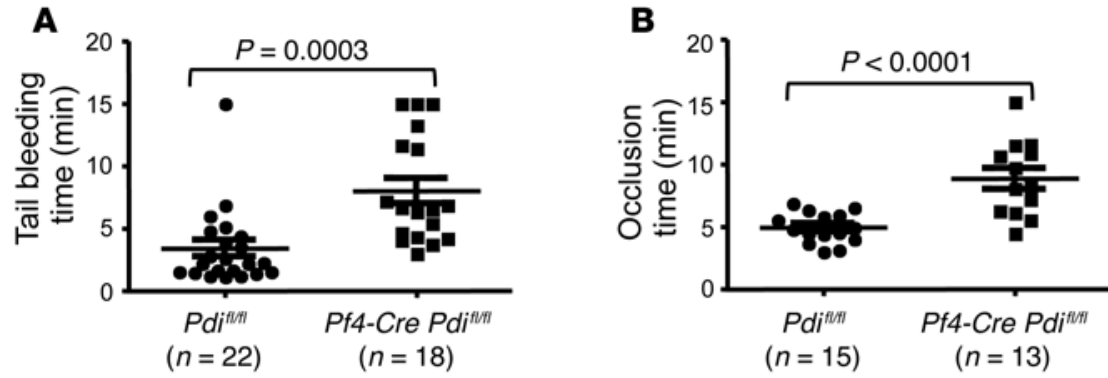

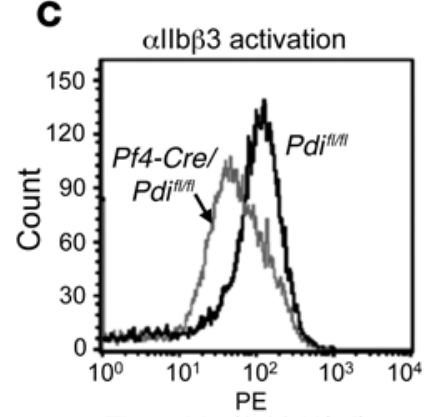

Thrombin $(0.02 \mathrm{U} / \mathrm{ml})$

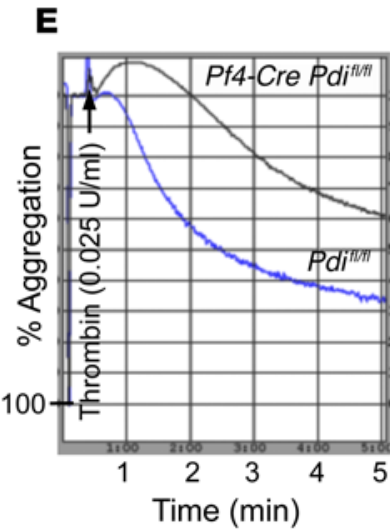

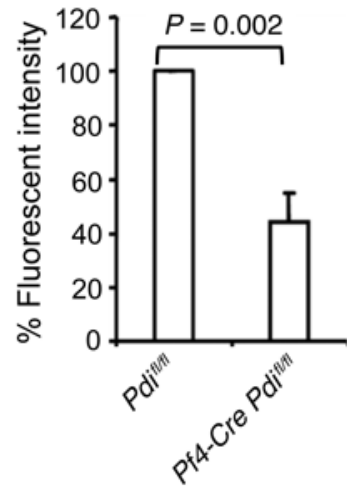

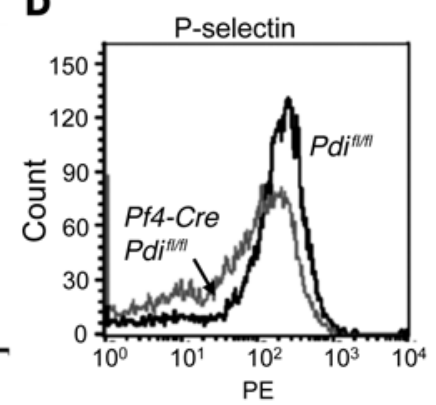

Thrombin $(0.02 \mathrm{U} / \mathrm{ml})$

$\mathbf{F}$

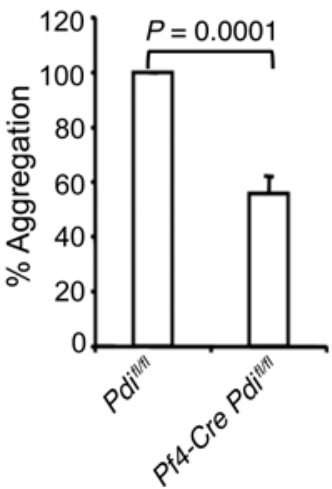

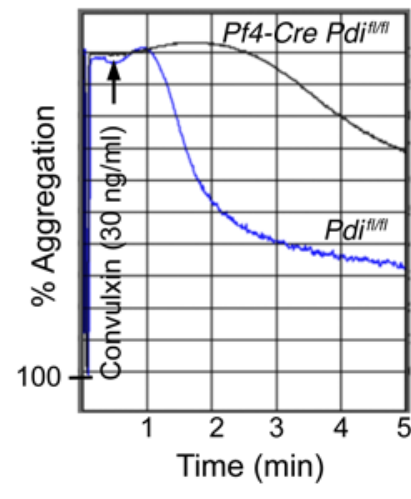

Figure 2. Deficiency of platelet PDI prolongs tail bleeding time and time to occlusion and impairs activation of $\alpha$ llb $\beta 3$, $P$ selectin expression, and platelet aggregation. (A) Tail bleeding times and (B) time to occlusion of $\mathrm{FeCl}_{3}$-induced carotid artery thrombosis in Pdifl/fl littermate controls compared with Pf4-Cre Pdifl/fl mice; mean $\pm \mathrm{SEM}, t$ test. (C) PDI-deficient platelets have a defect in activation of $\alpha$ llb $\beta 3$ by thrombin $(0.2 \mathrm{U} / \mathrm{ml}$; detected by the JON/A activation-dependent antibody). A representative tracing and cumulative data are shown; mean $\pm \mathrm{SEM}, n=4, t$ test. (D) Representative sample and combined results for the defect in thrombin-induced $(0.02 \mathrm{U} / \mathrm{ml}) \mathrm{P}$ selectin expression in platelets lacking PDI; mean $\pm \mathrm{SEM}, n=4, t$ test. Representative aggregation tracings and combined results $\pm \mathrm{SEM}$, showing the aggregation defect in PDI-deficient platelets activated with $(E)$ thrombin $(0.025 \mathrm{U} / \mathrm{ml})$ (mean $\pm \mathrm{SEM}, n=5, t$ test) and (F) convulxin (30 $\mathrm{ng} / \mathrm{ml}$ ) (mean $\pm \mathrm{SEM}, n=5, t$ test).

(Figure 5B). These results imply that the C-terminal active site of PDI has a role in hemostasis and thrombosis. Similar to that in the PDI-deficient platelets, thrombin-induced activation of the $\alpha \operatorname{IIb} \beta 3$ receptor, $\mathrm{P}$ selectin expression, and thrombin- and convulxininduced aggregation were decreased in platelets with PDI lacking the C-terminal active site (Figure 5, C-F). Higher doses of thrombin or convulxin overcame the aggregation defect (Supplemental Figure 4).

Incorporation of transgenic PDI(ss-oo) platelets into a growing thrombus is decreased. We examined incorporation of transgenic PDI(ss-oo) platelets in the transgenic mouse into a developing thrombus using $\mathrm{FeCl}_{3}$ in a mesenteric artery thrombosis model, in which the thrombosis is dependent on $\alpha \operatorname{IIb} \beta 3$ (36). Incorporation of PDI(ss-oo) platelets into the thrombus was substantially decreased (Figure 6A), with the decrease being statistically significant over the 5- to 15-minute time interval (Figure 6B). The aggregation defect of platelets from transgenic PDI(ss-oo) mice was corrected by the addition of rPDI(oo-ss) (Figure 6C). These data affirm the importance of the C-terminal CGHC motif of PDI in platelet aggregation and imply a role for this motif of PDI in platelet accumulation.

Recovery of platelet accumulation in mice lacking platelet PDI by infusion of rPDI(oo-ss). All cells in the transgenic PDI(ss-oo) mouse model, including cells in the vascular endothelial cells, express PDI with an inactive C-terminal CGHC motif. To determine the importance of the C-terminal active site of plateletderived PDI, we examined platelet accumulation using 
A
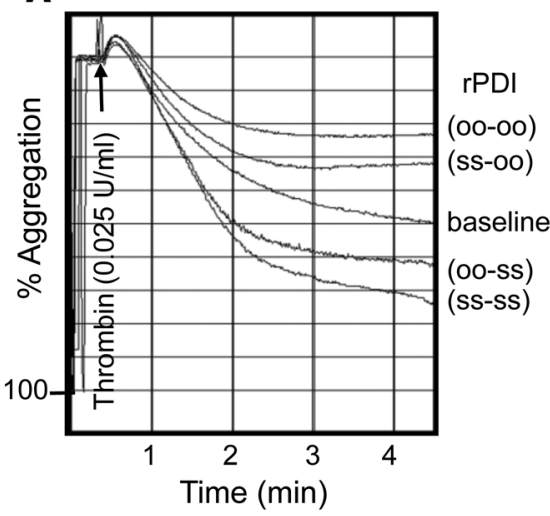

B

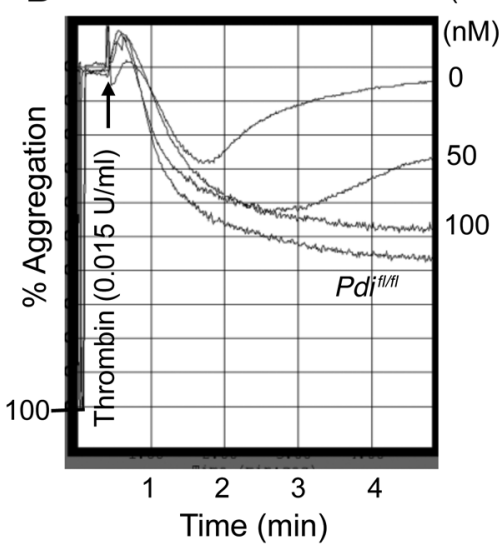

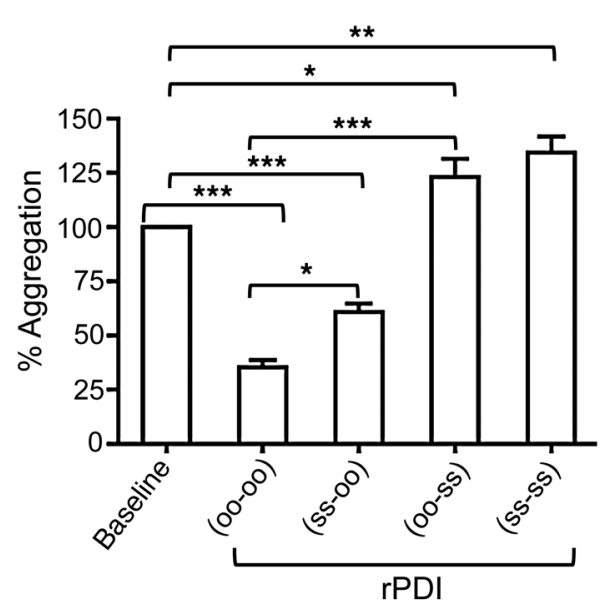

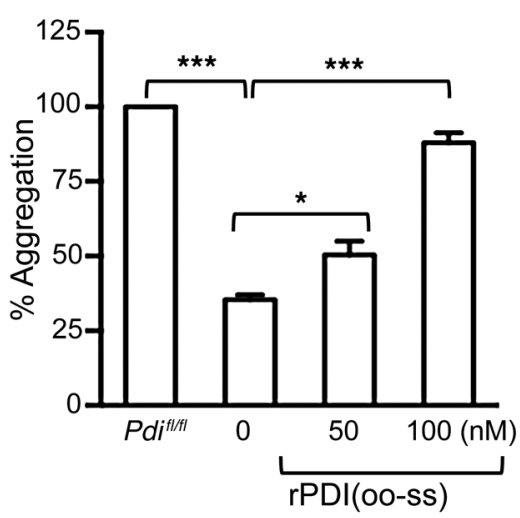

Figure 3. The C-terminal active site of PDI is critical for platelet aggregation. (A) Effect of preincubating washed human platelets $\left(2 \times 10^{8}\right.$ platelets $/ \mathrm{ml}$ ) with the recombinant $\mathrm{PDI}$ mutants [rPDI(ss-oo) and rPDI(oo-ss)]. Wild-type rPDI [rPDI(ss-0o)] and completely inactivated recombinant PDI [rPDI(00-00)] were used as controls. Submaximal aggregation (baseline) was stimulated with thrombin $(0.025 \mathrm{U} / \mathrm{ml})$. Representative tracings from a single experiment show the effect of adding PDI (1.5 $\mu \mathrm{M})$, along with corresponding cumulative data; mean $\pm \mathrm{SEM}$,

$n=5,{ }^{*} P<0.05,{ }^{* *} P<0.01,{ }^{* *} P<0.001$, ANOVA. (B) Correction of the aggregation defect of Pf4-Cre Pdifl/fl platelets by addition of rPDI(oo-ss). Aggregation of washed PDI-null mouse platelets preincubated with $\mathrm{rPDI}(00-\mathrm{ss})$ at the indicated concentrations is compared with aggregation of platelets from control Pdifl/fl mice. Representative tracings show the effect of adding $\mathrm{rPDI}(00-\mathrm{ss})$, along with corresponding cumulative data; mean \pm SEM, $n=5,{ }^{*} P<0.05$, ${ }^{* *} P<0.001$, ANOVA. $\mathrm{rPDI}$ at $37^{\circ} \mathrm{C}$ was added 5 minutes prior to the addition of thrombin $(0.015 \mathrm{U} / \mathrm{ml})$.

$\alpha$-granules (Figure 2 and Figure 5), we used eptifibatide to determine whether PDI-mediated P selectin expression was dependent on $\alpha \operatorname{IIb} \beta 3$. As expected, eptifibatide inhibited binding of an activation-specific antibody, JON/A, to $\alpha \operatorname{IIb} \beta 3$ (Figure 8A); however, it did not inhibit $\mathrm{P}$ selectin expression (Figure 8B). When we

Pf4-Cre Pdiflfl mice in the mesenteric artery model. Incorporation of PDI-deficient platelets into the thrombus was substantially decreased (Figure 6D), with the decrease being statistically significant over the 5- to 15-minute time interval (Figure 6E). These results indicate a role for platelet-derived PDI in platelet accumulation. Infusion of $\mathrm{rPDI}(\mathrm{oo}-\mathrm{ss})$ into the platelet-specific PDI-null mice rescued the defect in platelet accumulation (Figure 6, D and E), supporting the importance of the C-terminal CGHC motif of platelet PDI in platelet function in vivo.

$\alpha I I b \beta 3$ is the principal binding site for PDI on the platelet surface. To further understand the mechanism by which PDI regulates platelet aggregation and activation of $\alpha \operatorname{IIb} \beta 3$, we examined binding of PDI to $\alpha$ IIb $\beta 3$. PDI binding to thrombin-activated $\beta 3$-null platelets was substantially decreased compared with binding to wild-type platelets (Figure 7), as previously described (6). To study PDI binding to activated $\alpha \operatorname{IIb} \beta 3$ in the absence of platelet activation, we treated the platelets with $\mathrm{Mn}^{2+}$, which directly induces conformational changes in $\alpha \operatorname{IIb} \beta 3$ without causing platelet activation (42). Binding of PDI to $\mathrm{Mn}^{2+}$-treated $\beta 3$-null platelets was substantially decreased compared with binding to wild-type platelets (Figure 7, D-I). Both rPDI(oo-ss) (Figure 7, D-F) and rPDI(ss-oo) (Figure 7, G-I) bound similarly to $\mathrm{Mn}^{2+}$-treated platelets. These results are consistent with PDI binding to its substrate through the substrate-binding domain independently of the active site sequences (25).

The C-terminal CGHC motif of PDI is required for $P$ selectin expression and ATP secretion. To explore the regulatory mechanisms by which PDI mediates $\mathrm{P}$ selectin expression from added rPDI(ss-oo) or rPDI(oo-ss) to PDI-null platelets prior to platelet activation, only rPDI(oo-ss) recovered P selectin expression (Figure $8 \mathrm{C}$ ). In addition, eptifibatide inhibited binding of the activation-specific $\alpha \operatorname{IIb} \beta 3$ antibody, PAC1, to human platelets but did not inhibit P selectin expression (Supplemental Figure 5, A and B). rPDI(ss-oo) inhibited P selectin expression of human platelets (Supplemental Figure 5C). Together, these data indicate that the C-terminal active site of PDI mediates P selectin expression in a manner that is independent of $\alpha \operatorname{IIb} \beta 3$.

ATP secretion from dense granules of PDI-null platelets was about $50 \%$ of that of normal platelets (Figure $8 \mathrm{D}$ ). With addition of rPDI(oo-ss), but not rPDI(ss-oo), ATP secretion returned to normal, while aggregation remained unchanged. Using human platelets with maximal aggregation, rPDI(ss-oo) selectively inhibited ATP secretion but not aggregation (Supplemental Figure 5E). Taken together, these data imply that the C-terminal active site of PDI mediates ATP release and that the role of PDI in ATP secretion can be dissociated from its role in aggregation.

Intravascular PDI is required for fibrin deposition. Since each thrombosis model has advantages and disadvantages, in studying a novel mechanism, it is important to use more than one model to exclude a finding being model based. To this end, we used a laser-induced injury as our second thrombosis model. This model also allowed us to follow fibrin deposition simultaneously with platelet accumulation. We next examined the role of PDI in fibrin formation using Pf4-Cre Pdifl/ll mice, Mx1-Cre Pdillfl mice, and the transgenic PDI(ss-oo) mice. 


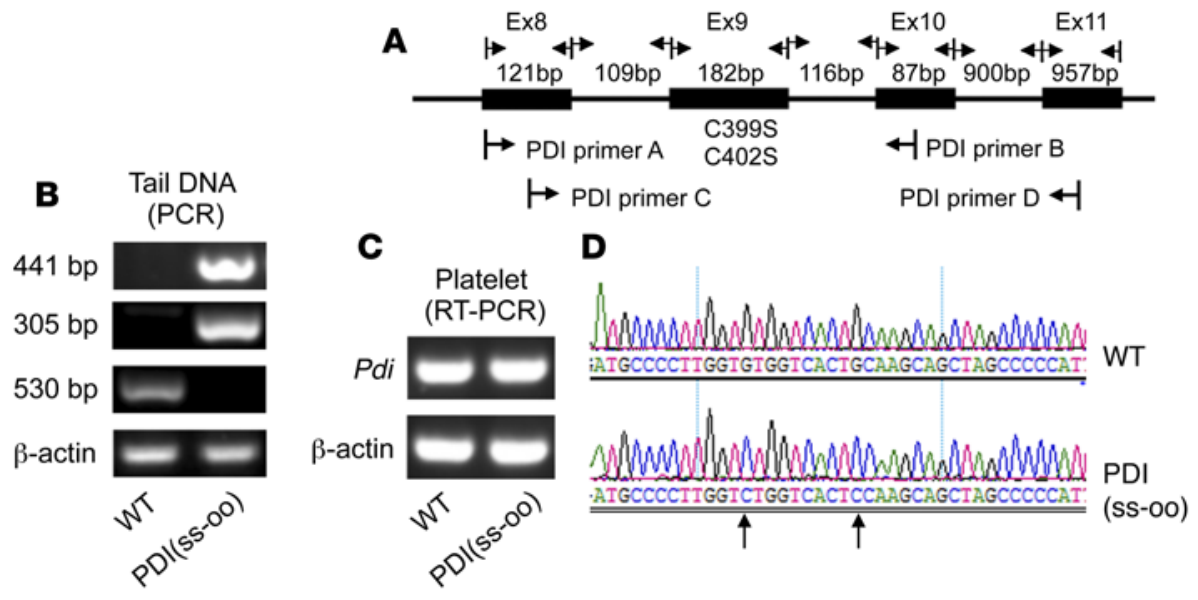

$\mathbf{E}$
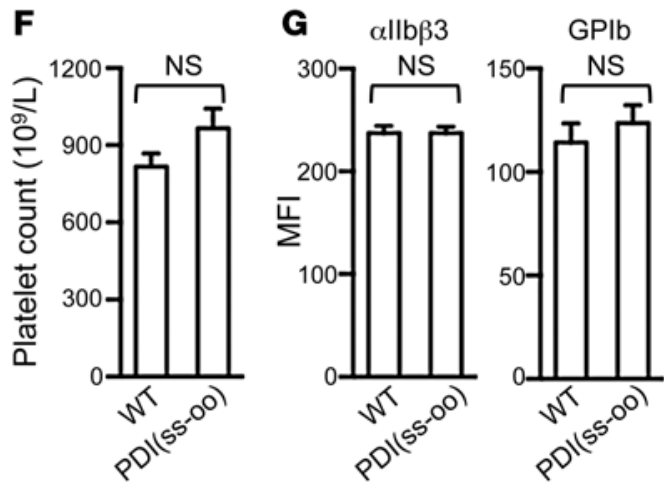

Figure 4. Characterization of knockout transgenic PDI(ss-oo) mice. (A) Primers used for PCR. (B) PCR products of tail DNA from wild-type mice and transgenic $P D I(s s-00)$ mice. PCR with primers $C$ and $D$ gave the expected 441-bp product (no product was seen from the wild-type allele, as this includes exons and is too large for PCR). PCR with primers A and B gave the predicted 305-bp product for the transgenic PDI(ss-oo) and the corresponding 530-bp product from the wild-type allele (includes both intron and exon elements). PCR products of $\beta$-actin served as control. (C) RT-PCR products of platelet mRNA from wild-type and transgenic PDI(ss-oo) mice. (D) Sequencing of cDNA prepared from platelet mRNA shows the $2 \mathrm{G}$-to- $C$ substitutions in the $C$-terminal active site domain. The $P C R$ product using primers $C$ and $D$ was subjected to sequencing using primer A. (E) Western blot of platelet lysate using a polyclonal rabbit anti-PDI antibody shows equal amounts of PDI, ERp57, and ERp72. The PLC $\gamma 2$ loading controls for PDI are shown. Separate loading controls were run for ERp57 and ERp72, with similar amounts of protein found in each sample (data not shown). Results in B-E are each representative of 3 separate experiments. (F) Platelet counts in wild-type and transgenic PDI(ss-oo) mice; mean $\pm \mathrm{SEM}, n=12$, $t$ test. (C) Normal expression of $\alpha$ llb $\beta 3$, GPIb, and GPVI in platelets from transgenic $\mathrm{PDI}(\mathrm{ss}-00)$ mice compared with wild-type mice; mean $\pm \mathrm{SEM}, n=10, t$ test.

While Pf4-Cre Pdit/f mice have a major defect in platelet accumulation at sites of injury, they did not have a defect in fibrin deposition (Figure 9, A-C, and ref. 6), suggesting that PDI from other cellular sources compensates for loss of platelet PDI. Previous studies have proposed that two major sources of extracellular PDI for thrombosis in vivo are platelets (6) and endothelial cells (16). To eliminate PDI contributions from other cells in the vascular system in addition to platelets, we generated $\mathrm{Mxl}$-Cre $\mathrm{Pdi} \mathrm{l}^{\mathrm{l} / \mathrm{l}}$ mice using an inducible promoter approach. PDI was dramatically reduced in platelets and endothelial cells of $\mathrm{MxI}$-Cre Pdifl/l mice after polyriboinosinic acid/polyribocytidylic acid [poly(I:C)] exposure (Supplemental Figure 6, A-E). PDI was also decreased in vessel wall fibroblasts, neutrophils, monocytes, and plasma of $\mathrm{Mx1}$-Cre Pdit/fl mice after poly(I:C) exposure (Supplemental Figure 6, F-I). ERp57

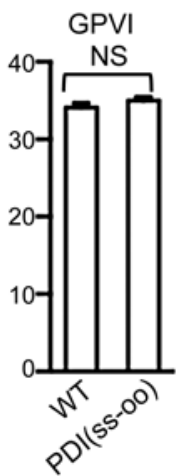

and ERp72 levels were unchanged in vessel wall cells and blood cells. Complete blood counts of Mx1-Cre Pdit/ll mice showed no difference from those of littermate controls also treated with poly(I:C). The platelet counts in both Mx1-Cre Pdit/fll and Pdit/fl littermate controls were comparable, although slightly lower than normal after poly(I:C) treatment (Supplemental Figure 7A), as previously described (43). Platelet size $(6.13 \pm 0.08 \mathrm{fl})$ was also comparable to that of $\mathrm{Cre}^{-}$littermates $(6.32 \pm 0.12, P=\mathrm{NS}, n=11$ per arm). There were no differences in expression of the major platelet surface glycoproteins, $\alpha \mathrm{IIb} 3, \mathrm{GpIb}$, and GpVI (Supplemental Figure 7B). The $\mathrm{PT}$ and the APTT of these mice were normal (Supplemental Figure 7C).

Mx1-Cre Pdil/f/ mice lacking PDI in vessel wall cells and platelets exhibited substantial defects in both platelet accumulation and fibrin deposition at sites of injury (Figure 9, D and E). The transgenic PDI(ss-oo) mice also had decreased platelet accumulation and a highly significant defect in fibrin deposition (Figure 10, A-C). Infusion of rPDI(oo-ss) containing only a functional C-terminal active site recovered platelet and fibrin deposition in both Mx1-Cre Pdit/fl mice and transgenic PDI(ss-oo) mice. These studies indicate that intravascular PDI mediates both platelet accumulation and fibrin deposition through the $\mathrm{C}$-terminal active site.

We previously demonstrated a role for the C-terminal active site of ERp57 in platelet accumulation and fibrin deposition $(36,37)$. To further distinguish the roles of PDI and ERp57 in thrombosis, we performed experiments in which we infused recombinant ERp57(ss-oo) with C-terminal CGHC motif inactivated [rERp57(ss-oo)] into the transgenic PDI(ss-oo) mice or into Mx1-Cre Pdif/ll mice. Infusion of rERp57(ss-oo) resulted in further inhibition of both platelet and fibrin deposition (Supplemental Figure 8, A-D). As we previously reported (37), Tie2-Cre $E R p 57^{f / f l}$ mice that lack ERp57 in endothelial cells and platelets had decreased platelet and fibrin accumulation (Supplemental Figure 8, E and F). Infusion of rPDI(ss-oo) into Tie2-Cre ERp5 $7^{f / f l}$ mice further inhibited platelet accumulation and fibrin deposition (Supplemental Figure 8, E and F). In contrast to the effect of infusing $\mathrm{rPDI}(\mathrm{oo}-\mathrm{ss})$ into $\mathrm{Mx1}-\mathrm{Cre} \mathrm{Pdi}^{\prime / l}$ mice (Figure 9, $\mathrm{E}$ and F), infusion of rERp57(oo-ss) into Mx1-Cre Pdil/fl mice did not result in significant recovery of platelet accumulation and fibrin 

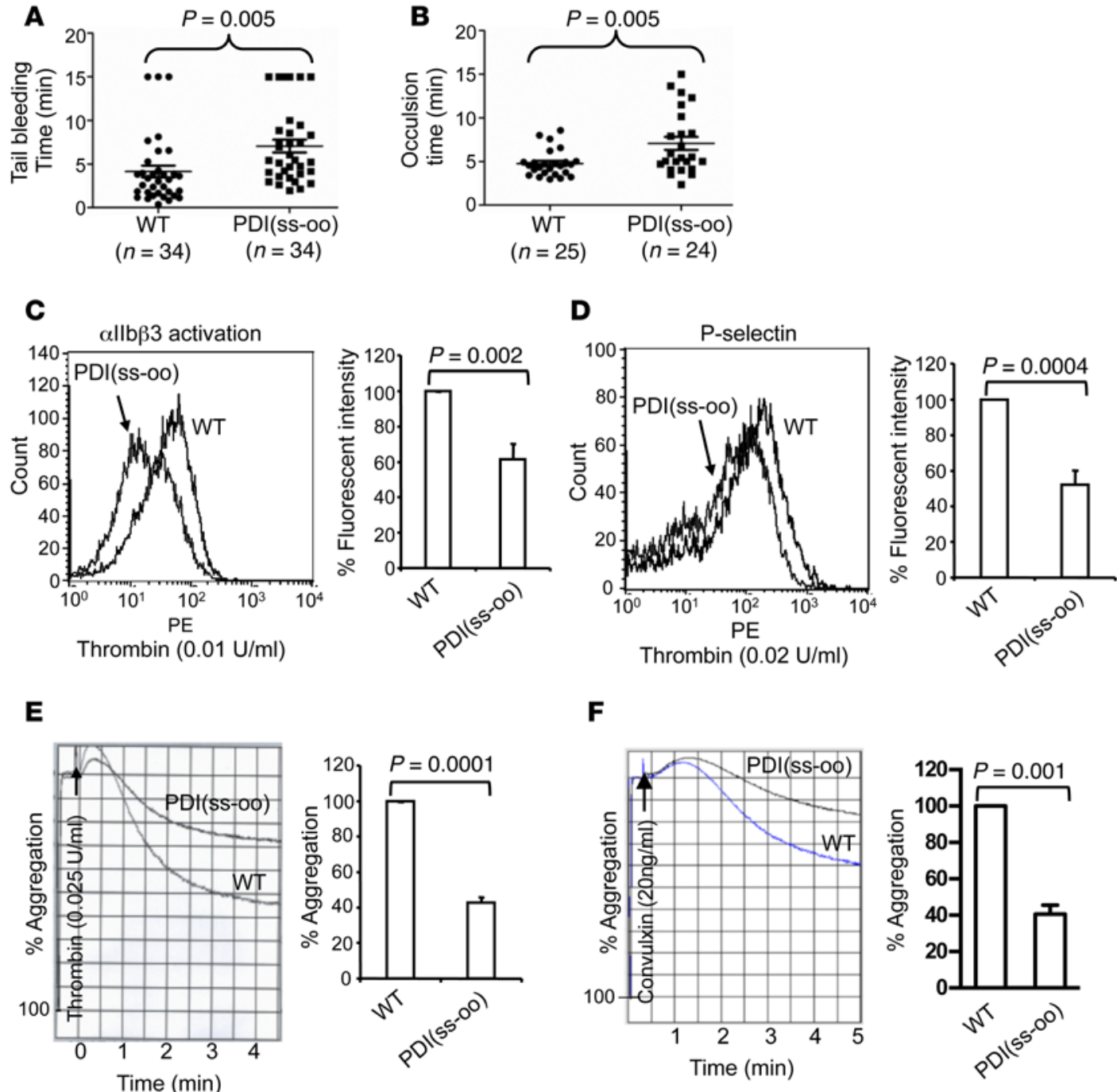

F
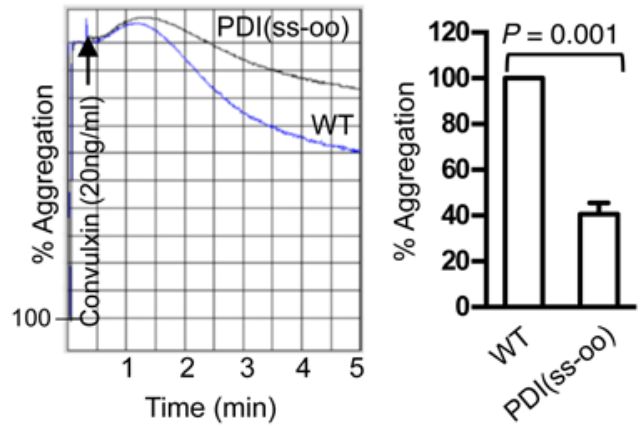

Figure 5. Transgenic PDI(ss-00) mice have impaired hemostasis, thrombosis, activation of $\alpha$ llb $\beta 3$, P selectin expression, and platelet aggregation. (A) Tail bleeding times and (B) times to occlusion of $\mathrm{FeCl}_{3}$-induced carotid artery thrombosis in transgenic $\mathrm{PDI}(\mathrm{ss}-00)$ mice compared with wild-type control mice; mean $\pm \mathrm{SEM}, t$ test. (C) Platelets from transgenic PDI(ss-00) mice have defects in thrombin-dependent $(0.02 \mathrm{U} / \mathrm{ml})$ activation of $\alpha$ llb $\beta 3$ (detected by the JON/A activation-dependent antibody). Representative flow histogram and cumulative data; mean \pm SEM, $n=6, t$ test. (D) $P$ selectin expression is decreased in thrombin-activated $(0.02 \mathrm{U} / \mathrm{ml})$ transgenic PDI(ss-0o) platelets. Representative histogram and cumulative data; mean $\pm \mathrm{SEM}, n=5, t$ test. Representative aggregation tracings and combined results of the aggregation defect in transgenic $\mathrm{PDI}(\mathrm{ss}-00)$ platelets using $(\mathrm{E})$ thrombin $(0.025 \mathrm{U} / \mathrm{ml})$ (mean $\pm \mathrm{SEM}, n=5, t$ test) and (F) convulxin ( $20 \mathrm{ng} / \mathrm{ml}$ ) (mean $\pm \mathrm{SEM}, n=4, t$ test).

deposition (Supplemental Figure 9, A and B). Similarly, infusion of rPDI(oo-ss) into Tie2-Cre ERp5 $7^{\text {f/ll }}$ mice did not result in significant recovery of platelet accumulation and fibrin deposition (Supplemental Figure 9, C and D). Together, these results suggest that the C-terminal active sites of ERp57 and PDI have distinct roles and that both are critical in these processes.

Since leukocytes may have a role in thrombosis (44) and PDI was decreased in the leukocytes of $\mathrm{Mx1}-\mathrm{Cre} P d i^{\natural / l}$ mice, we looked for the presence of leukocytes at the site of injury. Using in vivo labeling with FITC-conjugated anti-CD11b (44), we detected migrating leukocytes in the cremaster venules (Supplemental Video 1) but did not see rolling or adhesion of leukocytes at the sites of arteriolar injury in wild-type mice (Supplemental Video 2). Using Hoechst in vivo staining of leukocyte nuclei (45), leukocytes were visualized in the cremaster venules (Supplemental Video 3).
We again did not detect leukocyte rolling or adhesion at sites of arteriole injury (Supplemental Video 4). Therefore, leukocytes do not appear to be involved in our arteriole thrombosis model.

PDI accumulates at the site of vascular injury in 15 to $30 \mathrm{sec}-$ onds (15), and Kim et al. reported that PDI was found at the site of laser-induced vessel injury and on the developing platelet thrombus (6). Platelet thrombi consist of a heterogeneous population of platelets with varying degrees of activation, with the most activated platelets being in close proximity to the site of injury (46). Using confocal imaging, we visualized accumulation of Alexa Fluor 488-labeled rPDI(oo-ss) in wild-type and Pf4-Cre Pdifl/ mice (Supplemental Videos 5 and 6). PDI accumulated at the site of injury in close proximity to the developing platelet thrombus. In some areas, the PDI signal appeared to be on the developing thrombi, consistent with possible colocalization of PDI with platelets. 


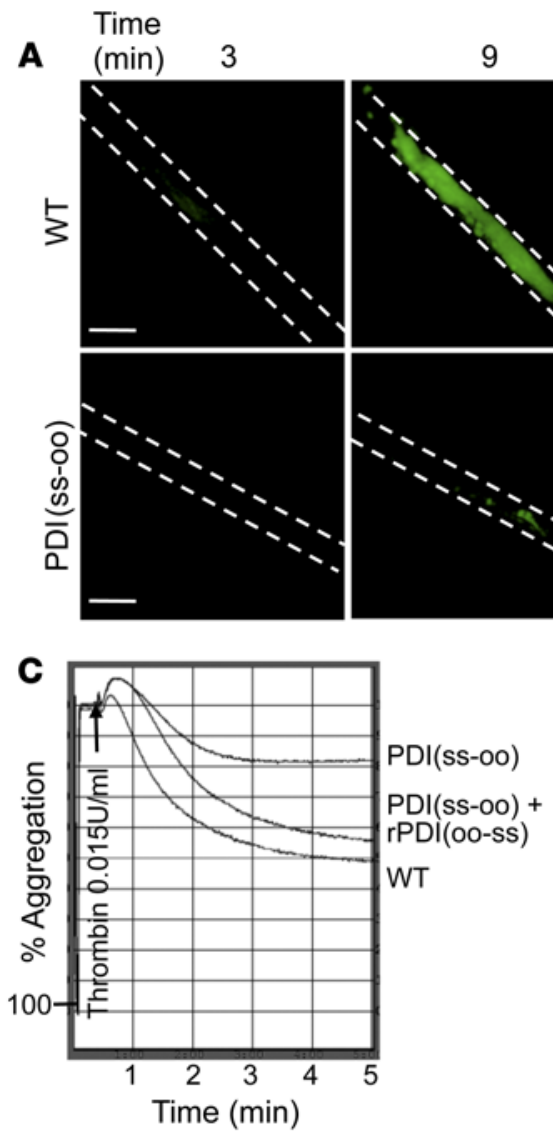

Time

D $(\min ) 3$
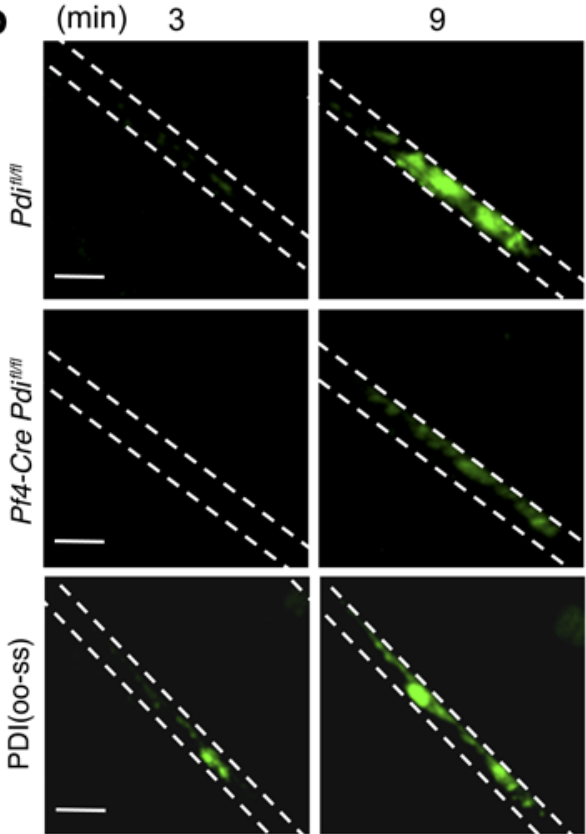

15
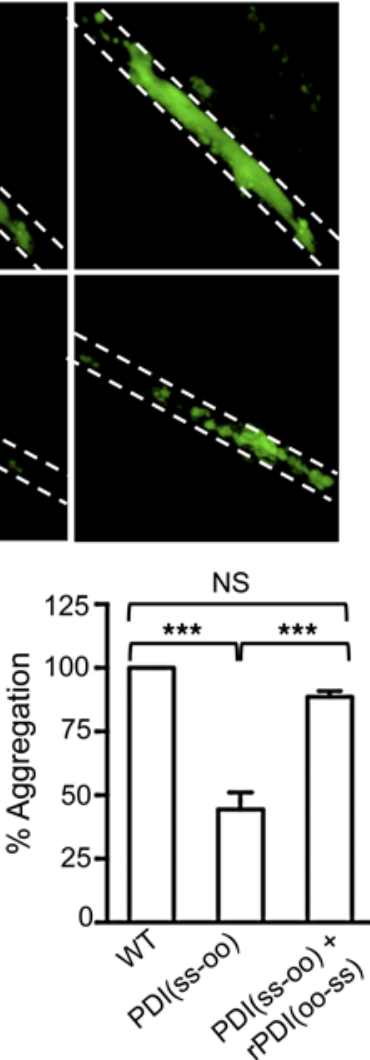

15
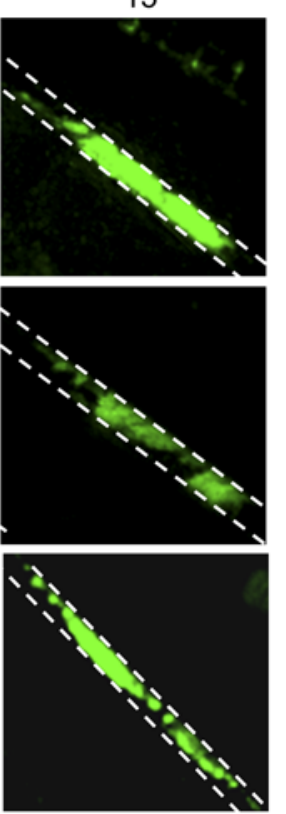
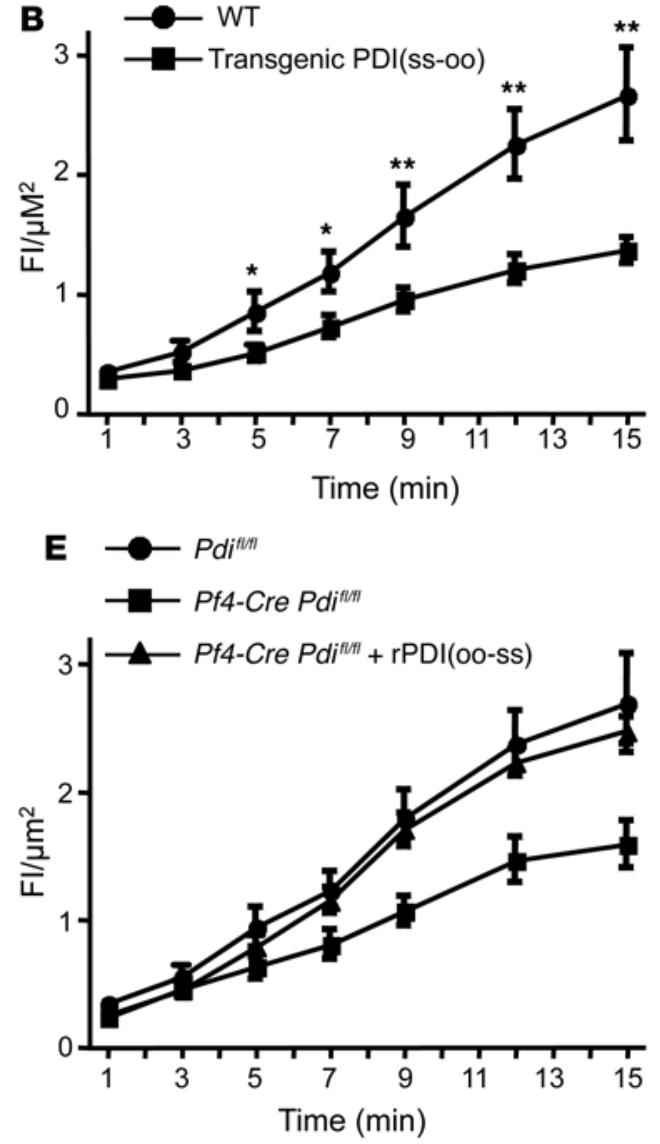

Figure 6. The C-terminal CGHC active site of $\mathrm{PDI}$ is required for platelet accumulation into a growing thrombus. ( $A$ and B) Incorporation of platelets into growing thrombi in wild-type and transgenic PDI(ss-0o) mice was detected by Alexa Fluor 488 anti-CD41 in $\mathrm{FeCl}_{3}$-induced mesenteric arterial injury. Mean artery diameters for wild-type mice are $121.6 \pm 4.5 \mu \mathrm{m}$, and those for transgenic PDI(ss-oo) mice are $118.0 \pm 6.7 \mu \mathrm{m}(P=\mathrm{NS})$. (A) Images at 3, 9, and 15 minutes are shown. Original magnification, $\times 100$. Scale bar: $100 \mu \mathrm{m}$. (B) FI per area analyzed $\left(\mathrm{Fl} / \mathrm{mm}^{2}\right)$ in wild-type ( $n=19$ from 7 mice) and transgenic PDI(ss-oo) ( $n=16$ from 6 mice) mice; mean $\pm \mathrm{SEM},{ }^{*} P<0.05,{ }^{* *} P<0.01$, $t$ test. (C) Correction of aggregation defect of transgenic $\mathrm{PDI}(\mathrm{ss}-\mathrm{0o})$ platelets by rPDI(oo-ss). Aggregation of platelets from wild-type mice and platelets from transgenic PDI(ss-00) mice preincubated with or without $100 \mathrm{nM} \mathrm{rPDI}(00-\mathrm{ss})$ for 5 minutes was induced by $0.015 \mathrm{U} / \mathrm{ml}$ thrombin. Representative tracings and combined results; mean $\pm \mathrm{SEM}, n=5$, ${ }^{* *} P<0.001$ ANOVA. (D and E) Recovery of platelet accumulation in Pf4-Cre $P d f^{f l / f l}$ mice by infusion of $\mathrm{rPDI}(\mathrm{oo}-\mathrm{ss})$. (D) Representative images at 3, 9, and 15 minutes after injury for $P d i^{f / f f l}$ control mice, Pf4-Cre $P d i^{f / f f l}$ mice, and Pf4-Cre Pdiflffl mice infused with rPDI(oo-ss) (250 $\mu \mathrm{g}$ per mouse). (E) Fl per area analyzed $\left(\mathrm{Fl} / \mathrm{mm}^{2}\right)$ in $P d i^{\mathrm{fl} / f l}$ mice ( $n=21$ from 8 mice); Pf4-Cre Pdifl/fl mice ( $n=18$ from 7 mice); and Pf4-Cre Pdifl/fl mice infused with rPDI(oo-ss) ( $n=16$ from 6 mice); mean $\pm \mathrm{SEM}$, ANOVA, Pdifl/fl versus Pf4-Cre Pdifl/fl: minute 5 , $P>0.05$; minute $7, P<0.05$; minute $9, P<0.01$; minute 12 , $P<0.01$; minute 15, $P<0.01$; Pf4-Cre Pdifl/fl plus rPDI(oo-ss) versus $P f 4$-Cre $P$ dif $^{f / f l}$ : minute $5, P>0.05$; minute $7, P<0.05$; minute $9, P<0.05$; minute 12, $P<0.05$; minute $15, P<0.05$. Mean artery diameters for $P d f^{f / f f l}$ mice are $118.8 \pm 4.7 \mu \mathrm{m}$, for Pf4-Cre Pdif//fl mice are $124.0 \pm 5.0 \mu \mathrm{m}$, and for Pf4-Cre Pdif/fl mice infused with rPDI(oo-ss) are $119 \pm 5.036 \mu \mathrm{m}(P=\mathrm{NS})$. Dotted lines mark the vessel wall. Original magnification, $\times 100$. Scale bar: $100 \mu \mathrm{m}$. 
A
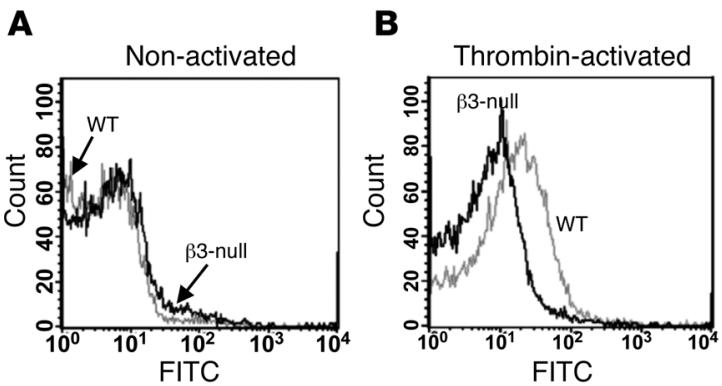

Thrombin $0.5 \mathrm{U} / \mathrm{ml}$
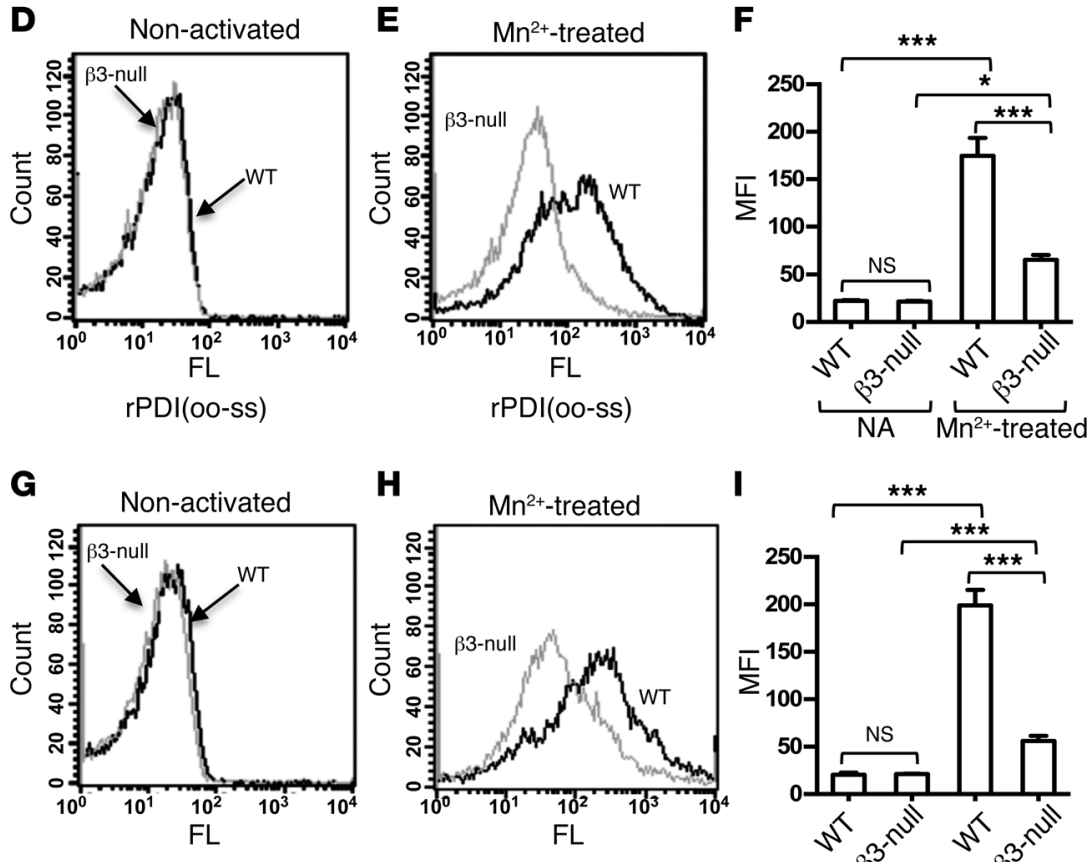

rPDI(ss-oo)
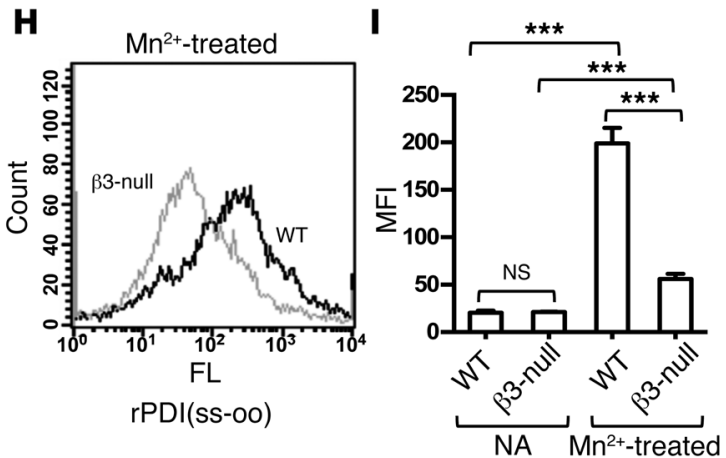

Figure 7. $P D I$ interacts with $\beta 3$ integrins on mouse platelets. Binding of Alexa Fluor 488 PDI to (A) nonactivated and (B) thrombin-activated wild-type and $\beta 3$-null mouse platelets. (C) Cumulative data for PDI binding to thrombin-activated platelets; mean $\pm \mathrm{SEM}, n=5,{ }^{* *} P<0.01,{ }^{* * *} P<0.001$, ANOVA. (D-F) Binding of Alexa Fluor 488-labeled rPDI(oo-ss) and (G-I) binding of Alexa Fluor 488labeled rPDI(ss-0o) to $\mathrm{Mn}^{2+}$-treated wild-type or $\beta 3$-null platelets. Washed mouse platelets $(3 \times$ $10^{8} / \mathrm{ml}$ ) were preincubated with Alexa Fluor 488 PDI $(30 \mu \mathrm{g} / \mathrm{ml})$ for 10 minutes at room temperature and then activated by thrombin $(0.5 \mathrm{U} / \mathrm{ml})$ or treated with $\mathrm{Mn}^{2+}(5 \mathrm{mM})$ for 5 minutes at room temperature. Surface binding of Alexa Fluor 488 $P D I$ was detected by flow cytometry; mean \pm SEM, $n=5,{ }^{*} P<0.05,{ }^{* *} P<0.001$, ANOVA.

Using two genetically engineered mouse models, we provide the first genetic evidence to our knowledge that intravascular PDI mediates fibrin generation and that it does this through its C-terminal active site. We found that $\mathrm{Mx1}$-Cre Pdillfl mice that did not express PDI in blood cells and vessel wall cells had a significant defect in fibrin formation. The importance of PDI in fibrin generation was confirmed in transgenic PDI(ss-oo) mice in which PDI function is defective. Reinhardt et al. showed that PDI infusion increased fibrin deposition in normal mice, demonstrating that PDI activated coagulation in vivo (13). Kim et al. subsequently showed that infusion of PDI increased both fibrin generation and platelet accumulation (6). Since PDI has a dual regulatory role in platelet accumulation and fibrin deposition and platelets can provide the pro-

To determine whether fibrin generation is dependent on platelets in our injury model, we infused eptifibatide to block platelet accumulation. As expected, platelet accumulation was entirely inhibited by eptifibatide (Figure 11, A and B). A major decrease in fibrin generation was also seen (Figure 11C), indicating that, in this model, platelets have a role in fibrin deposition. The decrease in fibrin deposition, but not the decrease in platelet accumulation, was reversed with infusion of wild-type recombinant PDI.

\section{Discussion}

Studies using a variety of in vitro assays and substrates over several decades indicate distinct biochemical activities for the two active sites of PDI (19-23). However, no study to date has addressed the relative importance of each active site of PDI in mammalian cells or a physiologic environment. Using genetically modified mouse models along with recombinant PDI variants, we demonstrate for the first time to our knowledge that the C-terminal active site of PDI is important for hemostasis and thrombosis. Whole-body knockout of PDI is lethal in embryogenesis, but the introduction of the PDI(ss-oo) transgene with a functional $\mathrm{N}$-terminal active site rescued the lethal phenotype, indicating that only the $\mathrm{N}$-terminal CGHC motif is important for murine survival. coagulant surface that supports coagulation (47), we examined whether the effect of PDI on coagulation was direct or secondary to its role in platelet accumulation. Infusion of eptifibatide decreased fibrin generation, indicating that platelet accumulation has a role in fibrin deposition. However, infusion of wild-type PDI into eptifibatide-treated mice recovered fibrin deposition without any recovery of platelet deposition (Figure 11), suggesting that PDI directly regulates fibrin generation. Furthermore, the defective fibrin deposition in the Mx1-Cre Pdillfl and transgenic PDI(ss-oo) mice was rescued by infusion of rPDI(oo-ss) (Figure 9F and Figure 10C), demonstrating the importance of the C-terminal active site of PDI for fibrin generation.

The C-terminal active site of PDI is also important for platelet function. Using human platelets, only PDI with a functional $\mathrm{C}$-terminal active site potentiated aggregation, and the aggregation defect in transgenic PDI(ss-oo) platelets was recovered by the addition of rPDI(oo-ss). Infusion of rPDI(oo-ss) corrected the platelet accumulation defect in Pf4-Cre Pdiflfl, Mx1-CrePdif/fl, and transgenic PDI(ss-oo) mice. Together, these data indicate that the catalytic activity of the C-terminal CGHC motif of PDI is critical for the conformational changes leading to activation of $\alpha \operatorname{IIb} \beta 3$ and platelet accumulation. 

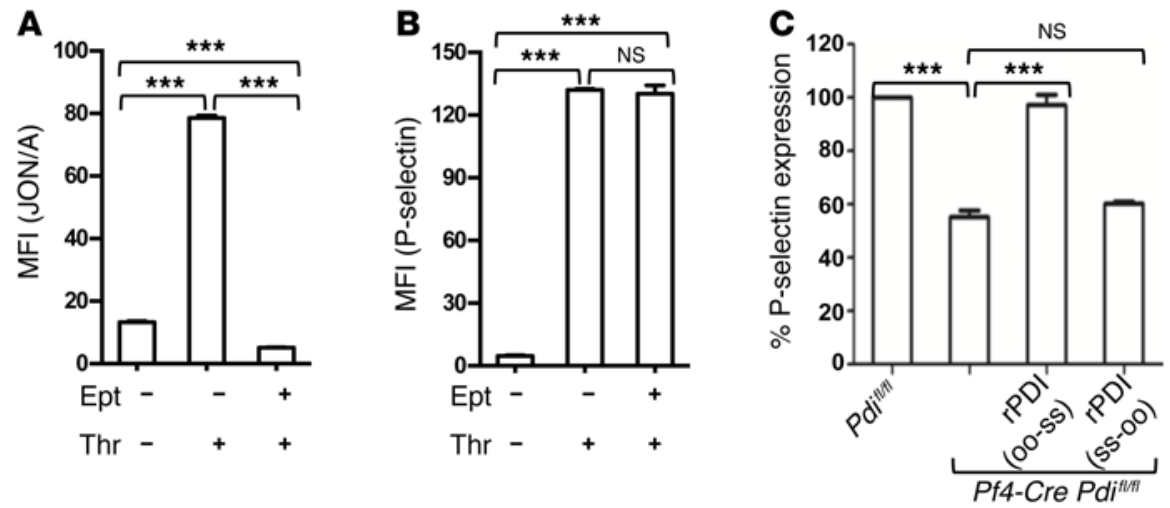

D
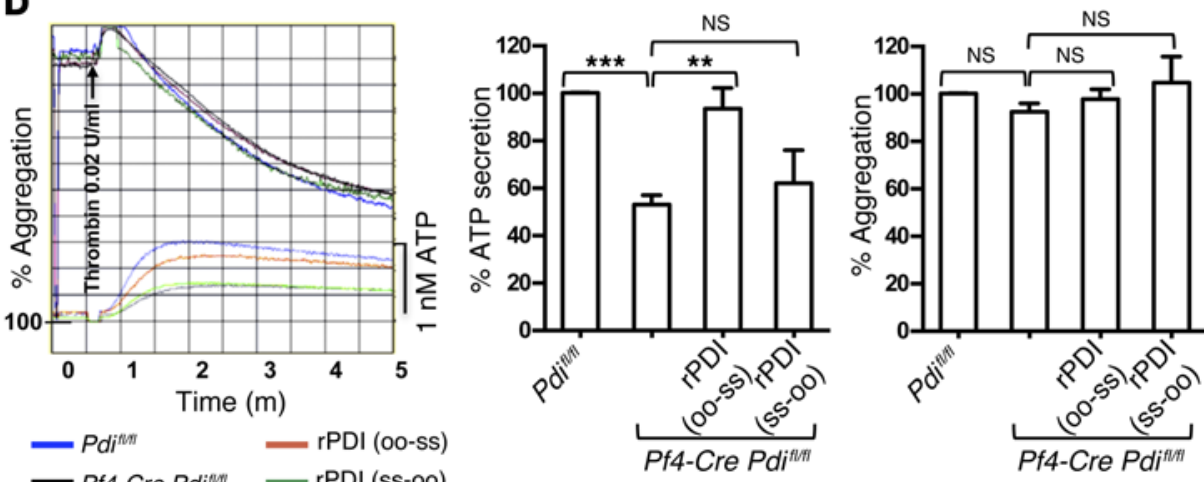

Figure 8. The C-terminal active site of PDI mediates $P$ selectin expression and ATP secretion from mouse platelets. (A) Eptifibatide (Ept) inhibits binding of the activation-specific antibody JON/A to $\alpha$ llb $\beta 3$ but (B) does not inhibit $P$ selectin expression $(n=3)$ in wild-type thrombin-activated (Thr-activated) platelets $(n=3)$; mean \pm SEM, ${ }^{* * *} P<0.001$, ANOVA. (C) Decreased P selectin expression in platelets from $P f 4-C r e P d i^{f / f l}$ mice compared with wild-type $\left(P d f^{f / / f l}\right)$ littermate mice is recovered by rPDI(0o-ss) but not rPDI(ss-0o) $(n=3)$. The platelets were activated with thrombin $(0.02 \mathrm{U} / \mathrm{ml})$, and $P$ selectin expression was measured by flow cytometry. (D) Deceased ATP secretion in platelets from Pf4-Cre Pdifl/fl mice compared with wild-type ( $\left.P d^{\prime f l / f l}\right)$ littermate mice is recovered by rPDI(oo-ss) but not $\mathrm{rPDI}(\mathrm{ss}-00)$. The platelets were activated with thrombin $(0.02 \mathrm{U} / \mathrm{ml})$, and aggregation and ATP secretion were monitored in the lumi-aggregometer. Representative aggregation tracings with ATP release and combined data, with $1 \mu \mathrm{M} \mathrm{rPDI(0o-ss)} \mathrm{or} \mathrm{rPDI(ss-0o)} \mathrm{added} \mathrm{to} \mathrm{some} \mathrm{samples} \mathrm{of} \mathrm{PDI-null} \mathrm{platelets.} \mathrm{In} \mathrm{the}$ experiments on ATP release, mean \pm SEM, $n=6$ [Pdifl/fl, Pf4-Cre Pdifl/fl, Pf4-Cre Pdifl/fl, and rPDI(oo-ss)], $n=3$ [Pf4-Cre Pdifl/fl and rPDI(ss-0o)]. ${ }^{* *} P<0.01,{ }^{* *} P<0.001$, ANOVA. Recombinant PDI was added 5 minutes prior to platelet activation.

The major binding site for PDI on the platelet surface appears to be $\alpha \operatorname{IIb} \beta 3$. The binding of PDI to thrombin-activated platelets was dependent on $\alpha \operatorname{IIb} \beta 3$. Since $\alpha \operatorname{IIb} \beta 3$ contributes to phospholipid exposure on the surface of activated platelets (48), the decreased binding of PDI to $\beta 3$-null platelets could be due to loss of non- $\alpha \operatorname{IIb} \beta 3$-binding sites. However, when $\alpha \operatorname{IIb} \beta 3$ on the platelet surface was directly activated with $\mathrm{Mn}^{2+}$, the binding of PDI was still dependent on $\alpha \operatorname{IIb} \beta 3$ (Figure 7). This suggests that PDI directly interacts with $\alpha \operatorname{IIb} \beta 3$. There was little binding of soluble PDI to resting platelets. Therefore, the increased binding of PDI to platelets is dependent on conformational changes that occur in $\alpha \operatorname{IIb} \beta 3$ as it undergoes activation. Since only rPDI(oo-ss) potentiates aggregation, the C-terminal active site of PDI may catalyze disulfide rearrangement in $\alpha \operatorname{IIb} \beta 3$, leading to the high-affinity form of this integrin and platelet aggregation.
The C-terminal active site of PDI mediates $P$ selectin expression and ATP secretion. Since P selectin expression was not dependent on outside-in signaling through $\alpha \operatorname{IIb} \beta 3$, the target for the $\mathrm{C}$-terminal active site of PDI on the platelet surface is evidently not this integrin. ATP secretion was similarly dependent on PDI with an intact C-terminal active site. Since normal aggregation was maintained in these experiments, the decrease in ATP secretion is consistent with the C-terminal active site of PDI regulating this process via a platelet surface target other than $\alpha \operatorname{IIb} \beta 3$. Both P selectin and ATP have a role in platelet function $(49,50)$ and are markers of $\alpha$ - and dense granule secretion, suggesting further mechanisms by which the $\mathrm{C}$-terminal active site of PDI mediates thrombosis.

We previously showed a role for ERp57 in thrombosis (37), and in the current studies, we provide evidence that PDI has a distinct role from ERp57 in thrombosis. Deficiency of PDI in Mx1-Cre Pdillfl mice with normal ERp57 levels resulted in defects in both platelet accumulation and fibrin generation (Figure 9, D-F), suggesting that ERp57 cannot compensate for the loss of PDI. Furthermore, when we infused rPDI(ss-oo) into Tie2-Cre ERp57f/fll mice or rERp57(ss-oo) into Mx1-Cre Pdi/f/l mice or the transgenic PDI(ss-oo) mice, we saw additional defects in platelet accumulation and fibrin deposition (Supplemental Figure 8). Moreover, Pf4-Cre Pdilfll mice had no defect in fibrin deposition (Figure 9C), whereas Pf4-Cre $E R p 57^{\Re / l}$ mice had a defect in fibrin deposition (37). Together, these data imply distinct roles of PDI and ERp57 in thrombosis.

In conclusion, we provide insights into the two CGHC redox -active sites in PDI. While the N-terminal site is required for murine viability, the C-terminal site is key for the role of intravascular PDI in platelet function and fibrin formation. In addition, we provide insights into the mechanisms by which PDI regulates thrombosis. Knowledge of the functional active site in thrombosis should allow for development of more specific and efficient anti-PDI reagents to maximize antithrombotic effects, while minimizing off-target effects. PDI molecules from different cellular sources form a spatial-temporal network necessary for initiation and maturation of thrombus formation. Both PDI and the highly homologous ERp57 $(36,37,41,51)$ regulate platelet function and fibrin formation, revealing the complexity of PDI 
A

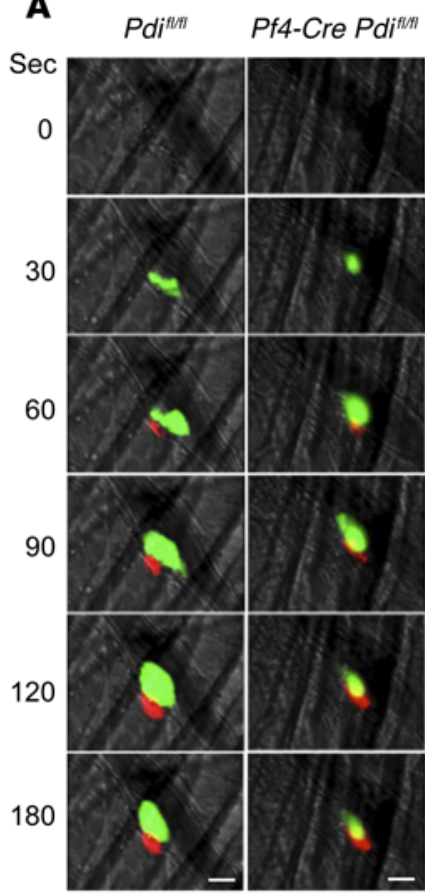

B
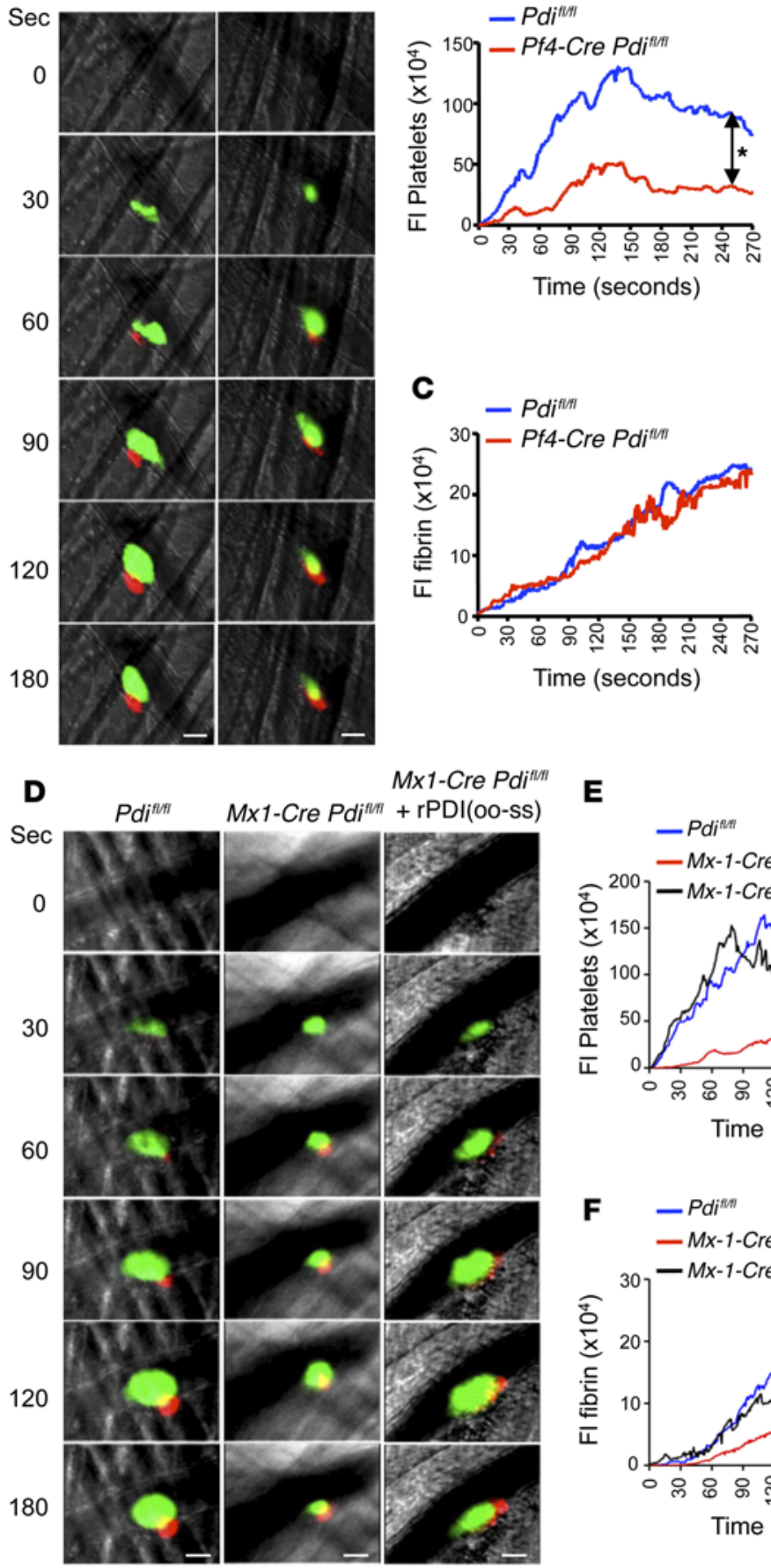

\section{E}
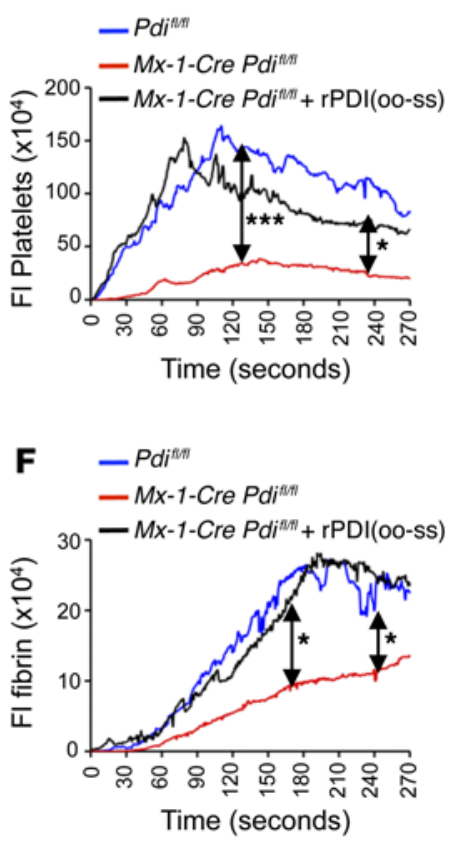

Figure 9. Intravascular PDI is required for fibrin deposition and platelet accumulation in vivo.

(A-C) Cremaster arteriole injury was induced in Pf4-Cre Pdifl/fl mice or Cre- Pdiflffl littermate control mice. Platelets and fibrin were detected using anti-CD41 F(ab) fragments (which bind to platelet $\beta 3$ ) conjugated to Alexa Fluor 488 and anti-fibrin antibody conjugated to Alexa Fluor 647. (A)

Representative intravital microscopy fluorescence images show platelet accumulation (green) and fibrin deposition (red) at selected time points up to 180 seconds after vascular injury. Original magnification, $\times 64$. Scale bar: $25 \mu \mathrm{m}$. The median integrated Fls of (B) anti-CD41 (platelet) and (C) anti-fibrin (fibrin) antibodies over 270 seconds. (D-F) Cremaster arteriole injury was induced in Mx1-Cre Pdifl/fl mice or Cre- Pdifl/fl littermate control mice. Mx1-Cre Pdifl/fl mice were infused with rPDI(oo-ss) (200 $\mu$ g per mouse). (D) Representative intravital microscopy fluorescence images show platelet accumulation (green) and fibrin deposition (red). Original magnification, $\times 64$. Scale bar: $25 \mu \mathrm{m}$. The median integrated Fls of $(\mathbf{E})$ antiCD41 (platelet) and (F) anti-fibrin (fibrin) antibodies. Fluorescence signal was not observed using fluorescently labeled control IgG (data not shown). The data were analyzed by area under curve, with a Mann-Whitney rank-sum test (37). Only significant differences are shown; ${ }^{*} P<0.05$; ${ }^{* *} P<0.001$.

The data were obtained from 30 thrombi in 3 mice for each experimental condition.

GpVI (Emfret); PE-conjugated anti-P selectin, allophycocyanin-conjugated anti-mouse CD144 (VE-cadherin) (clone eBioBV13), PE-conjugated anti-mouse CD31 (PECAM-1) (clone 390), PE-conjugated anti-mouse CD309 (Flk1) (clone Avas12a1), and labeled isotype-specific control antibodies (eBioscience); anti-PDI (RL90; Abcam); and anti-ERp5 (G-5) (Santa Cruz). Anti-fibrin antibody hybridoma (clone 59D8) was a gift from H. Weiler-Guettler (Blood Research Institute, Blood Center of Wisconsin, Milwaukee, Wisconsin, USA) (52). The following polyclonal rabbit antibodies were used: anti-PDI, anti-PLC $\gamma 2$, and anti-ERp5 (Santa Cruz) and anti-ERp72 (Stressgen). AntiCD41 F(ab)2 (BD Bioscience) was conjugated with Alexa Fluor 488 (Invitrogen). Additional materials included IRDye 800-conjugated goat anti-mouse IgG and IRDye 680-conjugated

family function in thrombosis. Further characterization of the specific reactions these enzymes catalyze should help advance our understanding of the mechanisms of thrombosis and also facilitate optimal development of antithrombotic agents, with dual inhibition of platelet function and coagulation.

\section{Methods}

Additional details are available in the Supplemental Methods.

Materials. The following monoclonal antibodies were used: PE-labeled JON/A, anti-mouse $\alpha$ IIb and $\beta 3$ complex, GpIb $\alpha$, and goat anti-rabbit IgG (LI-COR Bioscience); PVDF membrane (Millipore); $\alpha$-thrombin and quercetin-3-rutinoside (Sigma-Aldrich); poly(I:C) (R\&D Systems); eptifibatide (Shering Plough); and PDI (P4HB) mouse cDNA clone (MC200577) (OriGene Technologies). For studies of ATP secretion, purified $\alpha$-thrombin from J.W. Fenton II (Center for Laboratories and Research, New York State Department of Health, Albany, New York, USA) was used (53).

Generation and characterization of Pf4-Cre Pdit/fl mice with platelets deficient in PDI. The Pdi-floxed mice were generated by genOway. To generate conditional inactivation of the Pdi gene and megakaryocyte/ 
A

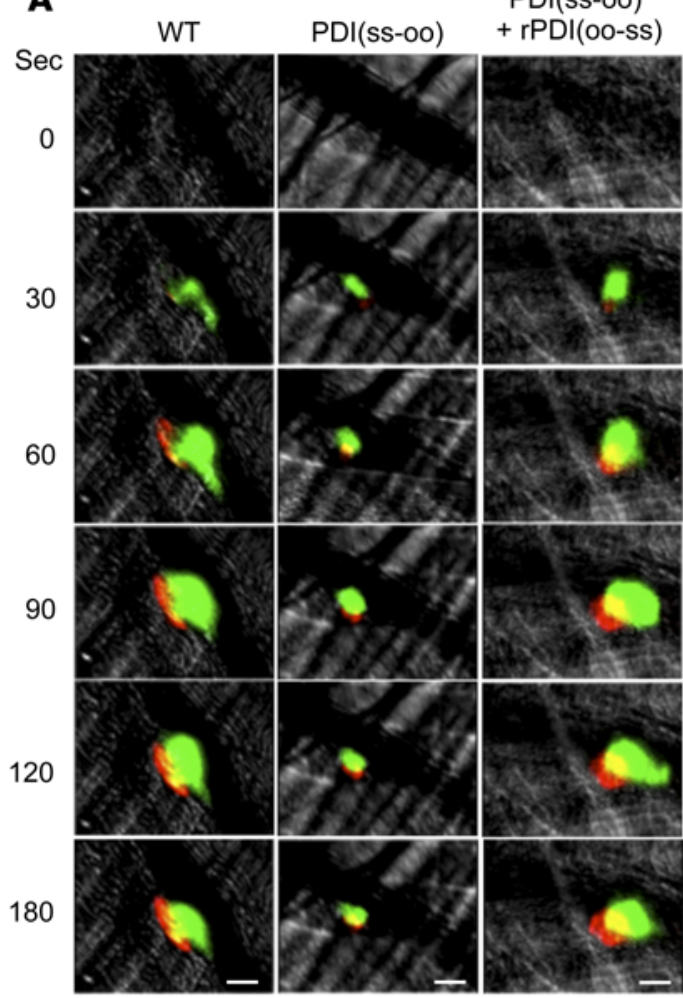

B
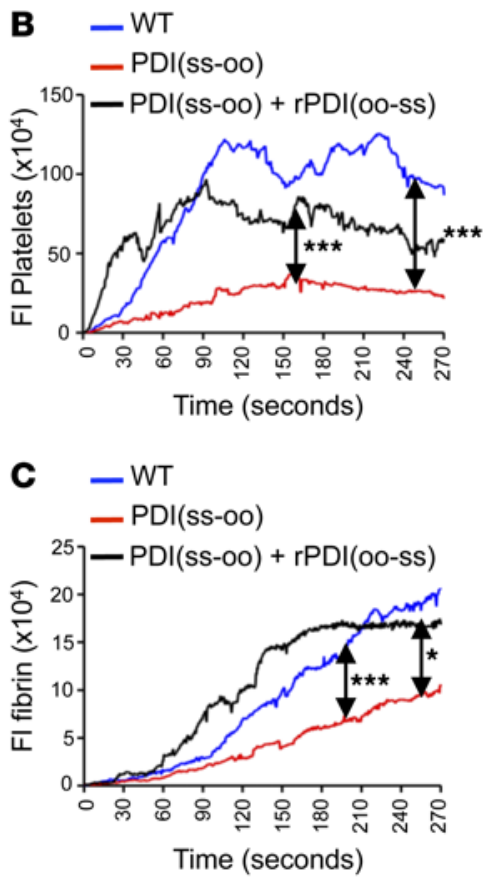

Figure 10. The C-terminal active site of PDI is required for fibrin deposition and platelet accumulation in vivo. Cremaster arteriole injury was induced in transgenic PDI(ss-0o) mice or wild-type controls. Transgenic PDI(ss-00) mice were infused with rPDI(00-ss) (200 $\mu$ g per mouse). Platelets and fibrin were detected as described in Figure 9. (A) Representative intravital microscopy fluorescence images show platelet accumulation (green) and fibrin deposition (red). Original magnification, $\times 64$. Scale bar: $25 \mu \mathrm{m}$. The median integrated Fls of (B) anti-CD41 (platelets) and (C) anti-fibrin (fibrin) antibodies. Data were analyzed as described for Figure 9, with only significant differences shown; ${ }^{*} P<0.05$; ${ }^{* * *} P<0.001$, Mann-Whitney rank-sum test. The data were obtained from 30 thrombi in 3 mice for each experimental condition.

observations). Therefore, we used an inducible $M x 1$-Cre model (43) to delete PDI in adult cells. MxI-Cre $P d i^{l / f l}$ mice were generated by mating Mx1-Cre mice (The Jackson Laboratory) with $P d i^{i / f l}$ mice. This produced Mx1-Cre Pdifl/fl mice and Pdi/f/ll litter-

platelet-specific deletion of PDI in mice, a targeting construct containing the first and second exons of the Pdi gene flanked by loxP sites (a 34-bp recognition sequence for Cre recombinase) and a PGK-neo selection cassette flanked by FRT sites (base pair sequence recognized by Flp recombinase) was generated (Figure $1 \mathrm{~A}$ ). Once the targeting construct was incorporated into the mouse germline, the neo cassette was removed by crossing the mice with mice expressing Flp recombinase (deleter mice) under a ubiquitous $\beta$-actin promoter (54). For confirmation of the recombination, the blotted genomic Pcil digest was tested using Southern blot analysis and an external 5'-SA-E-A probe. Mice homozygous for the Pdi floxed allele (loxP sites flanking exons 1 and 2) on a C57BL/6 × 129Sv background were crossed with Pf4-Cre mice (The Jackson Laboratory) on a C57BL/6 background (39). To generate platelet-specific $P d i$ knockout mice and experimental control mice, $P f 4$-Cre Pdifl/l mice were mated with $P d i^{f / l l}$ mice to yield Pf4-Cre $P d i^{f / l} / l$ mice and $P d i^{l / f l}$ littermate controls. Expression of platelet $P d i$ was assessed by RT-PCR of total platelet mRNA as previously described (36). Expression of Cre recombinase, ERp57, and $\beta$-actin was also assessed. The primers used were as follows: Pdi, sense $5^{\prime}$-tgacctggctcagcagtatg, antisense 5'-gaaaccgatgaccgtcactt; ERp57, sense 5 '-tatgatgggcctaggactgc, antisense 5 '-tgctggctgcttttaggaat; Cre, sense 5 '-ccatacagcacaccttttg, antisense 5 '-tgcacagtcagcaggtt; and $\beta$-actin, sense $5^{\prime}$-gtccctcacctcccaaaag, antisense 5 '-gctgcctcaacacctcaaccc.

All experiments with mice were performed on 8- to 12-week-old mice, with $50 \mathrm{mg} / \mathrm{kg}$ intraperitoneal pentobarbital used for anesthesia (41). Complete blood cells counts, white blood cell differential counts, and red blood cell and platelet indices were measured using a Sysmex Coulter Counter (XT2000-iV).

Generation and characterization of $M \times 1-C r e P d i^{f / f l}$ mice. The wholebody Pdi knockout is lethal (Y. Wu and D.W. Essex, unpublished mate controls. Pdi gene deletion was induced by intraperitoneal injection of $15 \mathrm{mg} / \mathrm{kg}$ poly(I:C) for 2 weeks every other day, as previously described $(43,55)$. Twelve days after the first injection, washed platelets were prepared from the treated $M x 1$-Cre Pdil/fl mice as described previously (36) to analyze inducible knockout gene deletion efficiency by Western blot. The laser-injury thrombosis experiments were performed 2 weeks after the last treatment with poly(I:C).

Isolation of endothelial cells from mice. Mouse endothelial cells were isolated from lungs and aortas as described previously (56). Briefly, after the experiments were completed, the mice were euthanized by exsanguination, followed by exposure of the thoracic cavity and removal of aorta. After the left atrium was cut, $10 \mathrm{ml}$ cold PBS with $0.1 \%$ heparinized solution was injected via the right ventricle to completely flush blood cells from the lungs. A needle with a polyethylene catheter was inserted into the left ventricle, and aorta was washed with cold PBS with 0.1\% heparinized solution using a pressurized pump. Lungs and aortas were removed and minced into small pieces, followed by incubation for 60 minutes at $37^{\circ} \mathrm{C}$ with $5 \mathrm{ml} 0.1 \%$ collagenase A (Roche Inc.). The digested tissue suspension was aspirated into to a $20-\mathrm{ml}$ syringe with a 14 -gauge cannula, and clumps were triturated into a single-cell suspension. The single-cell suspension was filtered through a $70-\mu \mathrm{m}$ strainer. The filtered cell suspension was centrifuged for 10 minutes at $300 \mathrm{~g}$, and the cell pellet was washed once with $0.5 \%$ BSA, 2 mM EDTA, and PBS (pH 7.2). The cell pellet was suspended with $90 \mu \mathrm{l}$ binding buffer per $10^{7}$ total cells, followed by the addition of $10 \mu \mathrm{l}$ CD31 MicroBeads (130-097-418, Miltenyi Biotec) per $10^{7}$ total cells. After incubation at $4^{\circ} \mathrm{C}$ for 15 minutes in the refrigerator, bead-bound cells were washed with $0.5 \%$ BSA, 2 mM EDTA, and PBS (pH 7.2) and removed using a magnetic EasySep (Stem Cell Technologies). Bead-bound cells were suspended in com- 


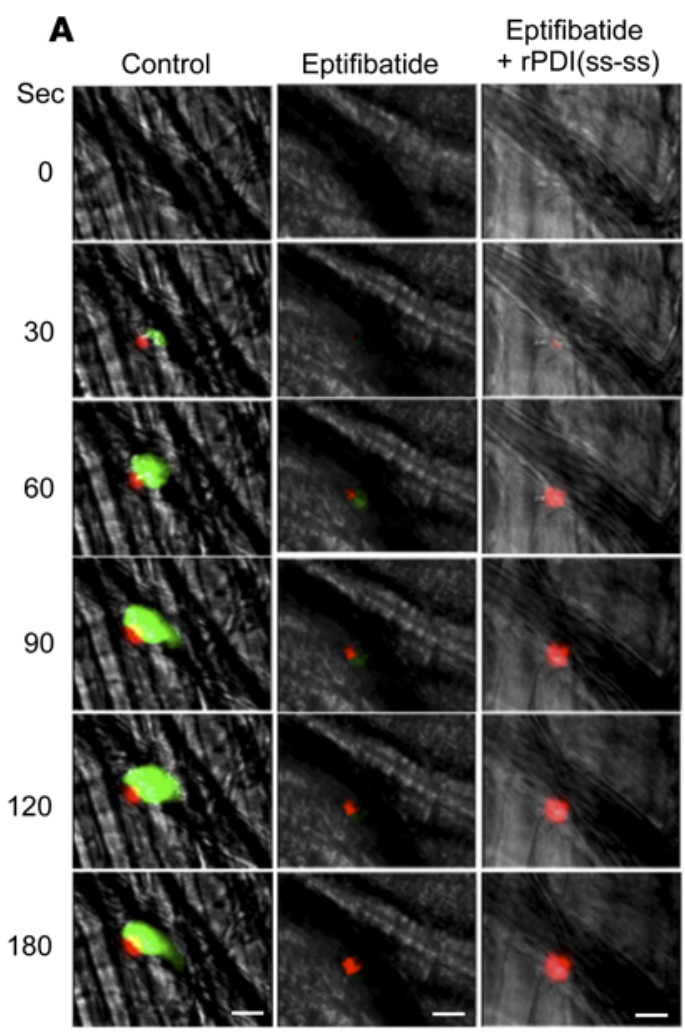

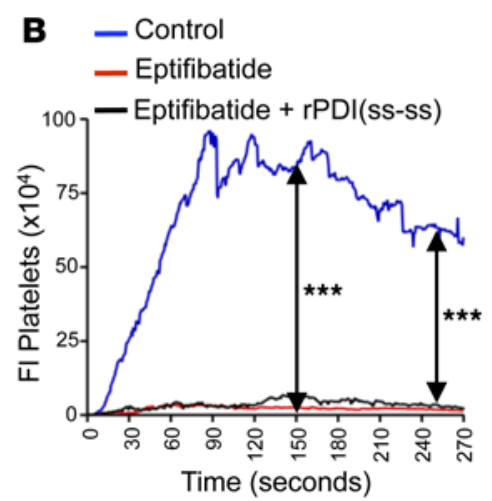

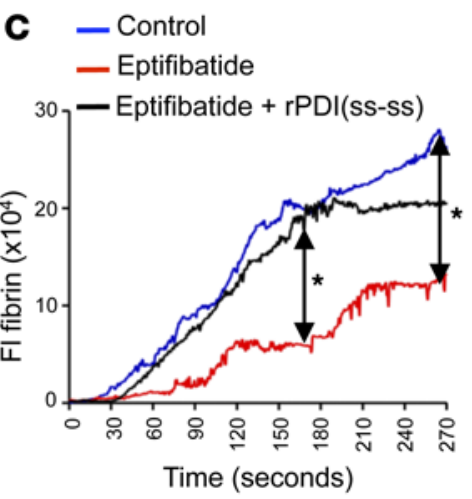

Figure 11. Infusion of rPDI(ss-ss) recovers fibrin deposition but not platelet accumulation in eptifibatide-treated mice. Eptifibatide ( $10 \mu \mathrm{g} / \mathrm{g}$ body weight) was infused into $\mathrm{C} 57 \mathrm{BL} / 6$ wild-type mice before injury and reinfused every 20 minutes for subsequent thrombi as described (37). Cremaster arteriole injury was induced in wild-type mice. In some experiments, rPDI(ss-ss) was infused (200 $\mu \mathrm{g}$ per mouse) 10 minutes prior to the first dose of eptifibatide. (A) Representative intravital microscopy fluorescence images show platelet accumulation (green) and fibrin deposition (red). Original magnification, $\times 64$. Scale bar: $25 \mu \mathrm{m}$. (B) Platelet accumulation and (C) fibrin deposition was analyzed as described for Figure 9, with only significant differences shown; ${ }^{*} P<0.05$; ${ }^{* *} P<0.001$, Mann-Whitney rank-sum test. The data were obtained from 30 thrombi in 3 mice for each experimental condition.

gous line was created by systematic breeding of heterozygotes and confirmed by quantitative PCR. Once the homozygous line was generated, homozygosity was maintained by inbreeding. In adopting this approach plete endothelial cell medium DMEM with 20\% FBS, 5\% microvascular growth supplement, and $0.1 \%$ gentamicin/amphotericin B and plated into a $100-\mathrm{mm}$ tissue culture dish coated with $0.1 \%$ collagen. CD $31^{+}$-selected cells were cultured for 3 to 7 days and fluorescently stained for VE-cadherin, Flk1, and CD31. The CD31- cells were collected and cultured in DMEM/F12 media containing 10\% FBS plus $1 \%$ penicillin-streptomycin. Cells were fluorescently stained for vimentin. The vimentin ${ }^{+}$cells were analyzed for PDI expression.

Generation of transgenic PDI(ss-oo) mice. Transgenic mice were generated using the transgene expressed in mice with a whole-body knockout as described previously (57). The PDI(ss-oo) mutant cDNA was generated by site-directed mutagenesis in wild-type mouse PDI cDNA, which was expressed in pCMV6-Neo plasmid (Origene) as a template $\left(\mathrm{C}^{399} \mathrm{GHC}^{402}\right.$ was substituted with $\left.\mathrm{S}^{399} \mathrm{GHS}^{402}\right)$. The CMV$\mathrm{PDI}(\mathrm{ss}-\mathrm{oo})$ plasmid was microinjected into C57BL/6 mouse embryos, which were implanted into pseudopregnant C57BL/6 mice. Transgenic PDI(ss-oo) mice were mated with heterozygous $C M V$-Cre Pdi ${ }^{\text {WT/fl }}$ mice. The resultant heterozygous $C M V$-Cre $P d i^{W T / f l}$ mice carrying the PDI(ss-oo) transgene were mated with heterozygous CMV-Cre Pdi ${ }^{\text {WT/fl }}$ mice to generate transgenic PDI(ss-oo) mice, whose endogenous $P d i$ was globally deleted (homozygous global Pdi knockout mice). These mice did not have wild-type Pdi alleles and exclusively expressed PDI(ss-oo). The expression of PDI(ss-oo) mRNA in platelets was tested by RT-PCR using the following primers: sense 5 '-tgcctgaagactgggacaa and antisense $5^{\prime}$-ccgctcgccattgtaatc.

To enhance the phenotype of these mice, we examined homozygotes for the transgene as described previously (57). However, when using PCR to genotype transgenic mice, we can reliably distinguish only heterozygous or homozygous for transgene or wild-type. To have a homogeneous population to study with full certainty, a homozy- of homozygous inbreeding, we did not have littermate controls. Control wild-type mice were obtained by breeding CMV-Cre Pdi ${ }^{\text {WT/fl }}$ mice on a mixed C57BL/6 $\times 129$ Sv background with wild-type C57BL/6 mice (The Jackson Laboratory). Therefore, the control and transgenic PDI(ss-oo) mice were on the same background.

RNA extraction, RT-PCR, and PCR. Total RNA was extracted from mouse platelets by using TRIzol Reagent (Life Technologies) according to the manufacturer's instructions. Reverse transcription was performed with total RNA (10 ng) using the RevertAid FirstStrand cDNA Synthesis Kit (Fermentas). The PCR reactions were carried out using cDNA obtained from reverse transcription or tail DNA using Dream Taq Green PCR Master Mix (Fermentas) according to the manufacturer's instructions.

Western blotting. Proteins from platelet lysates were separated by $8 \%$ SDS-PAGE and transferred onto a PVDF membrane (Millipore). The membranes were blocked with 1\% BSA in PBS. After extensive washing with TBS containing 0.1\% Tween 20, the immunoblots were incubated with the primary antibodies for 2 hours. Antibody binding was detected by using IRDye 800-conjugated goat anti-mouse IgG (LI-COR Bioscience) or IRDye 680-conjugated goat anti-rabbit IgG (LI-COR Bioscience) and visualized with an ODYSSEY infrared imaging system (LI-COR). The data are representative of 3 or 4 independent experiments.

Bleeding time analysis. The bleeding time assay was performed as described previously using a razor blade to transect the mouse tail $3 \mathrm{~mm}$ from the tip, with the tail immersed in a 12-ml test tube containing PBS at $37^{\circ} \mathrm{C}$ (41). Bleeding times were determined when the bleeding stopped for more than 10 seconds. When necessary, bleeding was stopped manually at the 15-minute time point. Age, sex, and body weight were comparable for the control and experimental groups. 
$\mathrm{FeCl}_{3}$-induced thrombosis of the carotid artery. Carotid artery thrombosis was induced in mice aged 6 to 8 weeks by treating the exposed artery with filter paper soaked in $5 \% \mathrm{FeCl}_{3}$ for 2 minutes. Blood flow through the carotid artery was monitored using a small animal transducer as described previously (41). An occlusion was considered stable when flow completely stopped for at least 5 minutes. The operator was blinded to the mouse genotype while performing experiments.

$\mathrm{FeCl}_{3}$-induced platelet accumulation and thrombosis of the mesenteric artery. To follow in vivo accumulation of platelets, Alexa Fluor 488-labeled anti-mouse CD $41 \mathrm{~F}(\mathrm{ab}) 2$ antibody was injected into mice through tail veins. The mesenteric artery was exteriorized through a midline abdominal incision. A single arteriole was visualized under a Zeiss Scope A fluorescent microscope. Vessel injury was generated by placing a $1-\times 2-\mathrm{mm}$ patch of Whatman No. 1 filter paper soaked in $5 \% \mathrm{FeCl}_{3}$ above the exposed artery for 1 minute. The filter paper was removed and platelet accumulation observed and photographed every minute for 15 minutes. The fluorescent intensity (FI) of the platelet accumulation and thrombus formation was analyzed by Image J software. The FI divided by the area analyzed gave the relative FI for each injury and is indicated by $\mathrm{FI} / \mu \mathrm{m}^{2}$. The area analyzed was obtained from the length analyzed multiplied by the diameter of each injured vessel. The length of the thrombus was from the beginning of the upstream edge of the thrombus to $1 \mathrm{~mm}$ downstream of this site. Background FI of the uninjured vessels was negligible. In some experiments, recombinant protein was intravenously injected into the mice 5 minutes before the injury.

Intravital microscopy of laser-induced thrombosis of the cremaster muscle arterioles. Laser-induced injury of the mouse cremaster muscle arteriole was performed as previously described $(37,46,58)$. Alexa Fluor 647-labeled anti-fibrin antibody and Alexa Fluor 488-labeled anti-CD $41 \mathrm{~F}(\mathrm{ab}) 2$ fragments (BD Biosciences) were infused at $0.1 \mu \mathrm{g} / \mathrm{g}$ into the jugular vein, followed by infusion of PDI mutants with BSA as a control (200 $\mu \mathrm{g}$ per mouse). After 5 minutes, arterioles (30- to $45-\mu \mathrm{m}$ diameter) were injured using a Laser Ablation system (Intelligent Imaging Innovations [I3]) with a Zeiss microscope (Axio Examiner D1) objective parfocal with the focal plane. The laser power was set to $55 \%$ to $65 \%$, and the laser was fired at the vessel wall with 1 to 3 pulses until thrombi were induced. Approximately 10 thrombi were studied in a single mouse. Injuries in which puncture of the vessel occurred or injuries in which no thrombi formed were excluded. The average number of laser pulses per injury was equal for all conditions (data not shown). Data were captured using a CCD camera (Cool Snap HQ2) using Slidebook 5.0 image acquisition and analysis software (I3). Data were collected for 5 minutes after vessel wall injury. Image analysis was performed using Slidebook Version 5.0 (I3). Data were obtained from a total of 30 thrombi per group for each experimental condition.

Flow cytometry and aggregation and secretion studies of mouse platelets. Flow cytometry studies of mouse platelets were performed using platelet-rich plasma prepared and diluted into Tyrode's buffer as described previously (41). The platelet-rich plasma was prepared from blood drawn into acid-citrate dextrose solution from the inferior vena cava (41). Aggregation studies were performed using washed platelets prepared as described previously (59). The monoclonal anti-PDI antibody RL90 and the IgG2a control antibody were extensively dialyzed against PBS to completely remove sodium azide, which may have an inhibitory effect on platelets. Platelet concentration was measured using a Sysmex Coulter Counter, and counts were adjusted to 200,000 platelets per $\mu 1$ for aggregation studies. Secretion of ATP was monitored with the Chronolog Lumi-Aggregometer as previously described (34).

Coagulation testing. The PT and the APTT were measured using a START 4 Coagulation Analyzer (Diagnostica Stago) according to the manufacturer's instructions. Mouse blood samples were collected

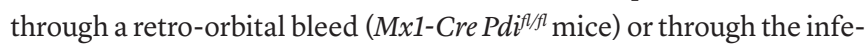
rior vena cava [PDI(ss-oo) mice] and combined with $147 \mathrm{mM}$ sodium citrate at a ratio of 9:1 and centrifuged at 3,000 $g$ for 10 minutes. For the Mx1-Cre Pdillfl mice, the samples were collected 1 week after the last treatment with poly(I:C). Plasma was incubated at $37^{\circ} \mathrm{C}$ for $2 \mathrm{~min}$ utes. The PT and the APTT were measured by adding NEOPLASTINE CI PLUS (Diagnostica Stago).

Flow cytometry and aggregation and secretion studies of human platelets. Flow cytometry studies of human platelets were performed using platelet-rich plasma prepared and diluted into Tyrode's buffer as described previously (41). Aggregation studies were performed using washed platelets prepared and suspended in modified Tyrode's buffer to which calcium (1 $\mathrm{mM}$ ) was added as described previously (60). The percentage of aggregation was calculated from the amplitude of the tracings at 5 minutes and normalized to the response of the untreated control within an individual experiment. Secretion of ATP was monitored with the Chronolog Lumi-Aggregometer as previously described (34).

Generation of recombinant human PDI protein. The cDNA for human PDI in the pTriEX-4 Neo vector (Novagen) with an N-terminal histidine tag was obtained from Bao-bing Zhu (University of Kansas Medical Center, Kansas City, Kansas, USA). After extraction from the gel, the resulting PCR product and the pTriEX-4 Neo vector were enzyme digested by SmaI and NotI. Human and mouse PDI were expressed in E. coli strain BL21 (DE3) pLysS (Promega) and purified on a Ni Sepharose High Performance column (GE Healthcare).

Preparation of catalytically inactive $\mathrm{N}$ - or C-terminal CGHC motifs of PDI. Two different mutant recombinant PDI enzymes were prepared (QuikChange Site-Directed Mutagenesis Kit, Stratagene) with both cysteine residues of one active site mutated to serine residues (C53S, C56S and C397S, C400S). These were designated as rPDI(oo-ss), representing the Cys/SH change to Ser/OH in the N-terminal CGHC active site), and rPDI(ss-oo), representing the $\mathrm{Cys} / \mathrm{SH}$ change to Ser/OH in the C-terminal CGHC active site. DNA sequencing confirmed the correct base substitutions. Recombinant wild-type PDI, designated as rPDI(ss-ss), and completely inactivated PDI (C53S, C56S, C397S, and C400S), designated as rPDI(oo-oo), were also prepared as previously described (41). rPDI(ss-ss) showed full activity in the Di-E-GSSG assay, while rPDI(oo-oo) showed no activity. The mutants with one functional active site showed intermediate activity between the wild-type PDI and the completely inactivated PDI.

Assay of enzyme-dependent disulfide reduction. PDI and ERp57 disulfide reductase activities against Di-E-GSSG were monitored in PDI assay buffer ( $0.1 \mathrm{M}$ potassium phosphate buffer, $2 \mathrm{mM}$ EDTA, $\mathrm{pH}$ 7.0) by adding ERp57 or PDI ( $20 \mathrm{nM})$ to Di-E-GSSG (150 nM) in the presence of $5 \mu \mathrm{M}$ DTT. The increase in fluorescence was monitored at $545 \mathrm{~nm}$, with excitation at $525 \mathrm{~nm}$, as described previously $(28,41)$. The degree of inhibition was relative to the total amount of EGSH formed over the time period of the assay or to the initial velocity of the reaction (nM EGSH formed per minute). 
Statistics. Data analysis was performed using the statistical software GraphPad Prism 5. For parametric comparison, the values were expressed as the mean \pm SEM, and 1-way ANOVA, followed by Tukey's test for multiple groups and 2-tailed Student's $t$ test for 2 groups were used. For nonparametric comparison of the area under the curve of the laser-injury experiments, Wilcoxon-Mann Whitney test was used (37). A $P$ value of less than 0.05 was considered significant.

Study approval. All studies on human platelets were performed after approval by the Temple University (IRB no. 20857) or the Soochow University (IRB no. 2012037) IRBs, with informed consent, in accordance with the Declaration of Helsinki. Experiments in mice were performed in accordance with the Temple University, Soochow University, and Children's Hospital of Philadelphia institutional guidelines and with approval of their IACUCs (IACUC no. 4211, Temple University; IACUC no. 001344, Soochow University; IACUC no. 12-000674, Children's Hospital of Philadelphia).

\section{Author contributions}

YW designed the project, supervised the research, analyzed the data, and helped revise the manuscript. JZ, YW, and LW performed research and collected and analyzed data. LR, VMH, and MP assisted with the in vivo hemostasis and thrombosis models, helped with manuscript writing and study design, and interpretation. DWE designed the project, supervised the research, analyzed the data, and wrote the manuscript.

\section{Acknowledgments}

This work was supported by start-up funds from Temple University School of Medicine, Priority Academic Program Development of Jiangsu Higher Education Institutions, Natural Science Foundation of China (81270592, 31201058, and 91539122), a VA Merit grant; and NIH grants (R01HL118526, to D.W. Essex, and P01HL110860, to M. Poncz).

Address correspondence to: Yi Wu, Cyrus Tang Hematology Center, Jiangsu Institute of Hematology, First Affiliated Hospital, Soochow University, 199 Ren-Ai Road, Suzhou, 215123, China. Phone: 512.65880877; E-mail: yiwu99@gmail.com. Or to: David W. Essex, Temple University School of Medicine, Rm. 204 MRB, 3420 North Broad St., Philadelphia, Pennsylvania 19140, USA. Phone: 215.707.6356; E-mail: david.essex@temple.edu.
1. Essex DW. Redox control of platelet function. Antioxid Redox Signal. 2009;11(5):1191-1225.

2. Essex DW, Li M. A polyclonal antibody to protein disulfide isomerase induces platelet aggregation and secretion. Thromb Res. 1999;96(6):445-450.

3. Lahav J, et al. Sustained integrin ligation involves extracellular free sulfhydryls and enzymatically catalyzed disulfide exchange. Blood. 2002;100(7):2472-2478.

4. Lahav J, Gofer-Dadosh N, Luboshitz J, Hess O, Shaklai M. Protein disulfide isomerase mediates integrin-dependent adhesion. FEBS Lett. 2000;475(2):89-92.

5. Lahav J, et al. Enzymatically catalyzed disulfide exchange is required for platelet adhesion to collagen via integrin $\alpha 2 \beta 1$. Blood . 2003;102(6):2085-2092.

6. Kim K, et al. Platelet protein disulfide isomerase is required for thrombus formation but not for hemostasis in mice. Blood. 2013;122(6):1052-1061.

7. Rao LV, Kothari H, Pendurthi UR. Tissue factor encryption and decryption: facts and controversies. Thromb Res. 2012;129(suppl 2):S13-S17.

8. Langer F, et al. Rapid activation of monocyte tissue factor by antithymocyte globulin is dependent on complement and protein disulfide isomerase. Blood. 2013;121(12):2324-2335.

9. Ahamed J, et al. Disulfide isomerization switches tissue factor from coagulation to cell signaling. Proc Natl Acad Sci U S A. 2006;103(38):13932-13937.

10. Pendurthi UR, Ghosh S, Mandal SK, Rao LV. Tissue factor activation: is disulfide bond switching a regulatory mechanism? Blood. 2007;110(12):3900-3908.

11. Jurk K, et al. Extracellular protein disulfide isomerase regulates feedback activation of platelet thrombin generation via modulation of coagulation factor binding. J Thromb Haemost. 2011;9(11):2278-2290.

12. Popescu NI, Lupu C, Lupu F. Extracellular protein disulfide isomerase regulates coagulation on endothelial cells through modulation of phosphatidylserine exposure. Blood. 2010;116(6):993-1001.

13. Reinhardt C, et al. Protein disulfide isomerase acts as an injury response signal that enhances fibrin generation via tissue factor activation. J Clin Invest. 2008;118(3):1110-1122.

14. Langer F, Ruf W. Synergies of phosphatidylserine and protein disulfide isomerase in tissue factor activation. Thromb Haemost. 2014;111(4):590-597.

15. Cho J, Furie BC, Coughlin SR, Furie B. A critical role for extracellular protein disulfide isomerase during thrombus formation in mice. JClin Invest. 2008;118(3):1123-1131.

16. Jasuja R, Furie B, Furie BC. Endotheliumderived but not platelet-derived protein disulfide isomerase is required for thrombus formation in vivo. Blood. 2010;116(22):4665-4674.

17. Jasuja R, et al. Protein disulfide isomerase inhibitors constitute a new class of antithrombotic agents. J Clin Invest. 2012;122(6):2104-2113.

18. Karala AR, Ruddock LW. Bacitracin is not a specific inhibitor of protein disulfide isomerase. FEBS J. 2010;277(11):2454-2462.

19. Vuori K, Myllylä R, Pihlajaniemi T, Kivirikko KI. Expression and site-directed mutagenesis of human protein disulfide isomerase in Escherichia coli. This multifunctional polypeptide has two independently acting catalytic sites for the isomerase activity. J Biol Chem. 1992;267(11):7211-7214.

20. Lyles MM, Gilbert HF. Mutations in the thioredoxin sites of protein disulfide isomerase reveal functional nonequivalence of the $\mathrm{N}$ - and C-terminal domains. J Biol Chem. 1994;269(49):30946-30952.

21. Darby NJ, Penka E, Vincentelli R. The multidomain structure of protein disulfide isomerase is essential for high catalytic efficiency. J Mol Biol. 1998;276(1):239-247.
22. Wang L, et al. Reconstitution of human Ero1Lalpha/protein-disulfide isomerase oxidative folding pathway in vitro. Position-dependent differences in role between the a and a' domains of protein-disulfide isomerase. J Biol Chem. 2009;284(1):199-206.

23. Westphal V, Darby NJ, Winther JR. Functional properties of the two redox-active sites in yeast protein disulphide isomerase in vitro and in vivo. JMol Biol. 1999;286(4):1229-1239.

24. Watanabe MM, Laurindo FR, Fernandes DC. Methods of measuring protein disulfide isomerase activity: a critical overview. Front Chem. 2014;2:73.

25. Hatahet F, Ruddock LW. Protein disulfide isomerase: a critical evaluation of its function in disulfide bond formation. Antioxid Redox Signal. 2009;11(11):2807-2850.

26. Essex DW, Li M, Feinman RD, Miller A. Platelet surface glutathione reductase-like activity. Blood. 2004;104(5):1383-1385.

27. Raturi A, Vacratsis PO, Seslija D, Lee L, Mutus B. A direct, continuous, sensitive assay for protein disulphide-isomerase based on fluorescence self-quenching. Biochem J. 2005;391(pt 2):351-357.

28. Raturi A, Mutus B. Characterization of redox state and reductase activity of protein disulfide isomerase under different redox environments using a sensitive fluorescent assay. Free Radic Biol Med. 2007;43(1):62-70.

29. Yan B, Smith JW. A redox site involved in integrin activation. J Biol Chem. 2000;275(51):39964-39972.

30. Essex DW, Li M, Miller A, Feinman RD. Protein disulfide isomerase and sulfhydryl-dependent pathways in platelet activation. Biochemistry. 2001;40(20):6070-6075.

31. Swiatkowska M, et al. Ero1 $\alpha$ is expressed on blood platelets in association with protein-disulfide isomerase and contributes to redox-controlled remodeling of $\alpha \operatorname{IIb} \beta 3$. J Biol Chem. 2010;285(39):29874-29883. 
32. Root P, Sliskovic I, Mutus B. Platelet cellsurface protein disulphide-isomerase mediated S-nitrosoglutathione consumption. Biochem J. 2004;382(pt 2):575-580.

33. Bell SE, Shah CM, Gordge MP. Protein disulfideisomerase mediates delivery of nitric oxide redox derivatives into platelets. Biochem J. 2007;403(2):283-288.

34. Essex DW, Li M. Protein disulphide isomerase mediates platelet aggregation and secretion. $\mathrm{Br} \mathrm{J}$ Haematol. 1999;104(3):448-454.

35. Flaumenhaft R, Furie B, Zwicker JI. Therapeutic implications of protein disulfide isomerase inhibition in thrombotic disease. Arterioscler Thromb Vasc Biol. 2014;35(1):16-23.

36. Wang L, et al. Platelet-derived ERp57 mediates platelet incorporation into a growing thrombus by regulation of the $\alpha \mathrm{IIb} \beta 3$ integrin. Blood. 2013;122(22):3642-3650.

37. Zhou J, et al. The disulfide isomerase ERp57 is required for fibrin deposition in vivo. J Thromb Haemost. 2014;12(11):1890-1897.

38. Walczak CP, Tsai B. A PDI family network acts distinctly and coordinately with ERp29 to facilitate polyomavirus infection. J Virol. 2011;85(5):2386-2396.

39. Tiedt R, Schomber T, Hao-Shen H, Skoda RC. Pf4-Cre transgenic mice allow the generation of lineage-restricted gene knockouts for studying megakaryocyte and platelet function in vivo. Blood. 2007;109(4):1503-1506.

40. Soldà T, Garbi N, Hämmerling GJ, Molinari M. Consequences of ERp57 deletion on oxidative folding of obligate and facultative clients of the calnexin cycle. J Biol Chem. 2006;281(10):6219-6226.

41. Wu Y, et al. The disulfide isomerase ERp57 mediates platelet aggregation, hemostasis, and throm- bosis. Blood. 2012;119(7):1737-1746.

42. Litvinov RI, et al. Functional and structural correlations of individual $\alpha I I b \beta 3$ molecules. Blood. 2004;104(13):3979-3985.

43. Weng Z, et al. PTEN regulates collagen-induced platelet activation. Blood. 2010;116(14):2579-2581

44. Darbousset R, et al. Tissue factor-positive neutrophils bind to injured endothelial wall and initiate thrombus formation. Blood. 2012;120(10):2133-2143.

45. Nishimura $S$, et al. In vivo imaging visualizes discoid platelet aggregations without endothelium disruption and implicates contribution of inflammatory cytokine and integrin signaling. Blood. 2012;119(8):e45-e56.

46. Stalker TJ, et al. Hierarchical organization in the hemostatic response and its relationship to the platelet-signaling network. Blood. 2013;121(10):1875-1885.

47. Lhermusier T, Chap H, Payrastre B. Platelet membrane phospholipid asymmetry: from the characterization of a scramblase activity to the identification of an essential protein mutated in Scott syndrome. J Thromb Haemost. 2011;9(10):1883-1891.

48. Reverter JC, et al. Inhibition of platelet-mediated, tissue factor-induced thrombin generation by the mouse/human chimeric 7E3 antibody. Potential implications for the effect of c7E3 Fab treatment on acute thrombosis and "clinical restenosis”. J Clin Invest. 1996;98(3):863-874.

49. Merten M, Thiagarajan P. P-selectin in arterial thrombosis. Z Kardiol. 2004;93(11):855-863.

50. Fung CY, Cendana C, Farndale RW, Mahaut-Smith MP. Primary and secondary agonists can use $\mathrm{P} 2 \mathrm{X}(1)$ receptors as a major pathway to increase intracellular $\mathrm{Ca}(2+)$ in the human platelet.
J Thromb Haemost. 2007;5(5):910-917.

51. Holbrook LM, et al. The platelet-surface thiol isomerase enzyme ERp57 modulates platelet function. J Thromb Haemost. 2012;10(2):278-288.

52. Weiler-Guettler H, et al. A targeted point mutation in thrombomodulin generates viable mice with a prethrombotic state. J Clin Invest. 1998;101(9):1983-1991.

53. Huang EM, Detwiler TC, Milev Y, Essex DW. Thiol-disulfide isomerization in thrombospondin: effects of conformation and protein disulfide isomerase. Blood. 1997;89(9):3205-3212.

54. Rodriguez CI, et al. High-efficiency deleter mice show that FLPe is an alternative to Cre-loxP. Nat Genet. 2000;25(2):139-140.

55. Yilmaz OH, et al. Pten dependence distinguishes haematopoietic stem cells from leukaemia-initiating cells. Nature. 2006;441(7092):475-482.

56. Lee S, Wurtzel JG, Singhal SS, Awasthi S, Goldfinger LE. RALBP1/RLIP76 depletion in mice suppresses tumor growth by inhibiting tumor neovascularization. Cancer Res. 2012;72(20):5165-5173.

57. Johnson AW, et al. Cognitive and motivational deficits together with prefrontal oxidative stress in a mouse model for neuropsychiatric illness. Proc Natl Acad Sci U S A. 2013;110(30):12462-12467.

58. Greene TK, et al. In vivo efficacy of plateletdelivered, high specific activity factor VIII variants. Blood. 2010;116(26):6114-6122.

59. Wu Y, et al. Role of Fc receptor gamma-chain in platelet glycoprotein Ib-mediated signaling. Blood. 2001;97(12):3836-3845.

60. Wu Y, et al. Interaction between von Willebrand factor and glycoprotein Ib activates Src kinase in human platelets: role of phosphoinositide 3-kinase. Blood. 2003;101(9):3469-3476. 